\title{
Recycler Electron Cooling project: Mechanical vibrations in the Pelletron and their effect on the beam
}

\author{
G. Kazakevich, A. Burov, C. Boffo, P. Joireman, \\ G. Saewert, C. W. Schmidt, and A. Shemyakin
}

\section{Introduction}

The Fermilab's Recycler ring will employ an electron cooler to cool stored 8.9 $\mathrm{GeV}$ antiprotons [1]. The cooler is based on an electrostatic accelerator, Pelletron [2], working in an energy-recovery regime. A full-scale prototype of the cooler has been assembled and commissioned in a separate building [3]. The main goal of the experiments with the prototype was to demonstrate stable operation with a $3.5 \mathrm{MeV}, 0.5$ A DC electron beam while preserving a high beam quality in the cooling section. The quality is characterized, first of all, by a spread of electron velocities in the cooling section, which may be significantly affected by mechanical vibration of the Pelletron elements. This paper describes the results of vibration measurements in the Pelletron terminal and correlates them with the beam motion in the cooling section.

\section{Setup description}

Some parameters of the electron cooling setup are listed in Tab. 1, and its mechanical schematic is shown in Fig. 1.

Table 1: Electron Cooling System Parameters

\begin{tabular}{|l|l|l|c|}
\hline Parameter & Cooler design value & $\begin{array}{l}\text { Working point of } \\
\text { the prototype }\end{array}$ & Units \\
\hline \hline Electrostatic Accelerator & \multicolumn{3}{|l|}{} \\
\hline Terminal Voltage & 4.34 & 3.5 & $\mathrm{MV}$ \\
Beam Current & 0.5 & 0.5 & $\mathrm{~A}$ \\
Terminal Voltage ripple, rms & 500 & $<500$ & $\mathrm{~V}$ \\
\hline Cooling Section & 20 & 75 & $\mathrm{~m}$ \\
\hline Length & 0.7 & $\mathrm{G}$ \\
Solenoid Field & $50-150$ & 6 & $\mathrm{nTorr}$ \\
Vacuum & 0.1 & 0.3 & $\mathrm{~mm}$ \\
Beam Radius & 6 & \multicolumn{2}{l}{} \\
Electron Angles, rms & $\leq 0.2$ &
\end{tabular}

The electron beam is generated by an electron gun and is accelerated in an acceleration tube of the Pelletron to the full energy. After passing through a transfer line, the beam is bent by $90^{\circ}$, and then it enters an $18 \mathrm{~m}$ long solenoid, a prototype of the cooling section. A $180^{\circ}$ bend downstream of the cooling section sends electrons through a 
return line toward the Pelletron, where the beam is decelerated in the second Pelletron tube and is absorbed in a collector at the kinetic energy of 3-5 keV.

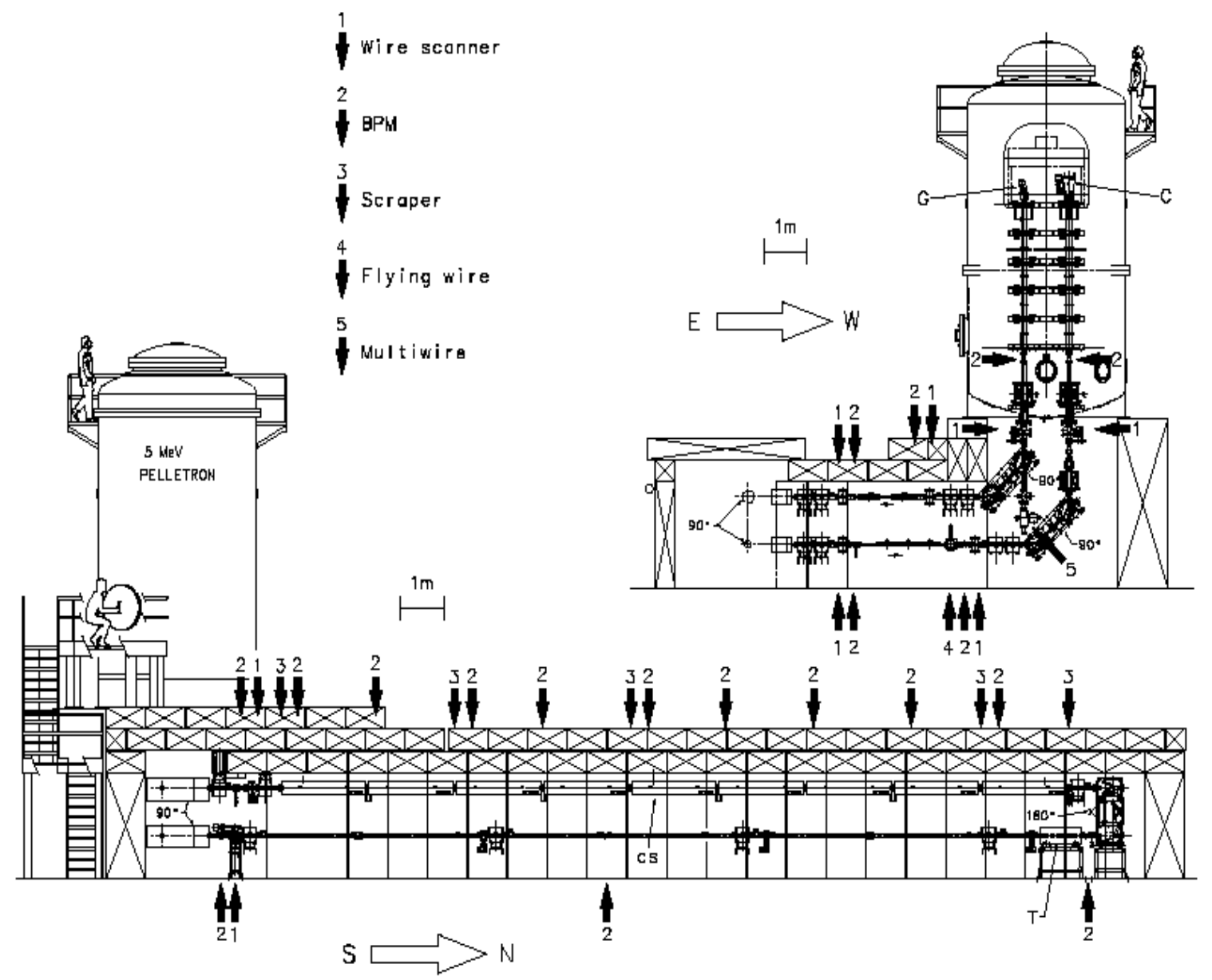

Fig. 1: Mechanical schematic of the setup. Arrows shows positions of various types of diagnostics. $90^{\circ}$ and $180^{\circ}$ label the corresponding bends. Letters indicate: G-gun, C-collector, CS- cooling section, T - quadrupole triplet. Arrows marked 2 show positions of BPMs.

The mechanical schematic of the Pelletron is shown in Fig. 2 and 3. The main parts of the Pelletron are a tank filled with an insulating gas and a column, where two metal-ceramic acceleration tubes are mounted. Typical working pressure of the $\mathrm{SF}_{6}$ insulating gas was 70 psi (5.8 bars of absolute pressure).

The column consists of several hollow aluminum disks called separation boxes, joined by six cylindrical supports (posts). In the area of acceleration, each post is a sandwich of bonded ceramic and metal discs. Hoops with a diameter equal to the outside diameter of the separation boxes $(\sim 1.8 \mathrm{~m})$ are mounted from the discs for electrostatic shielding. 


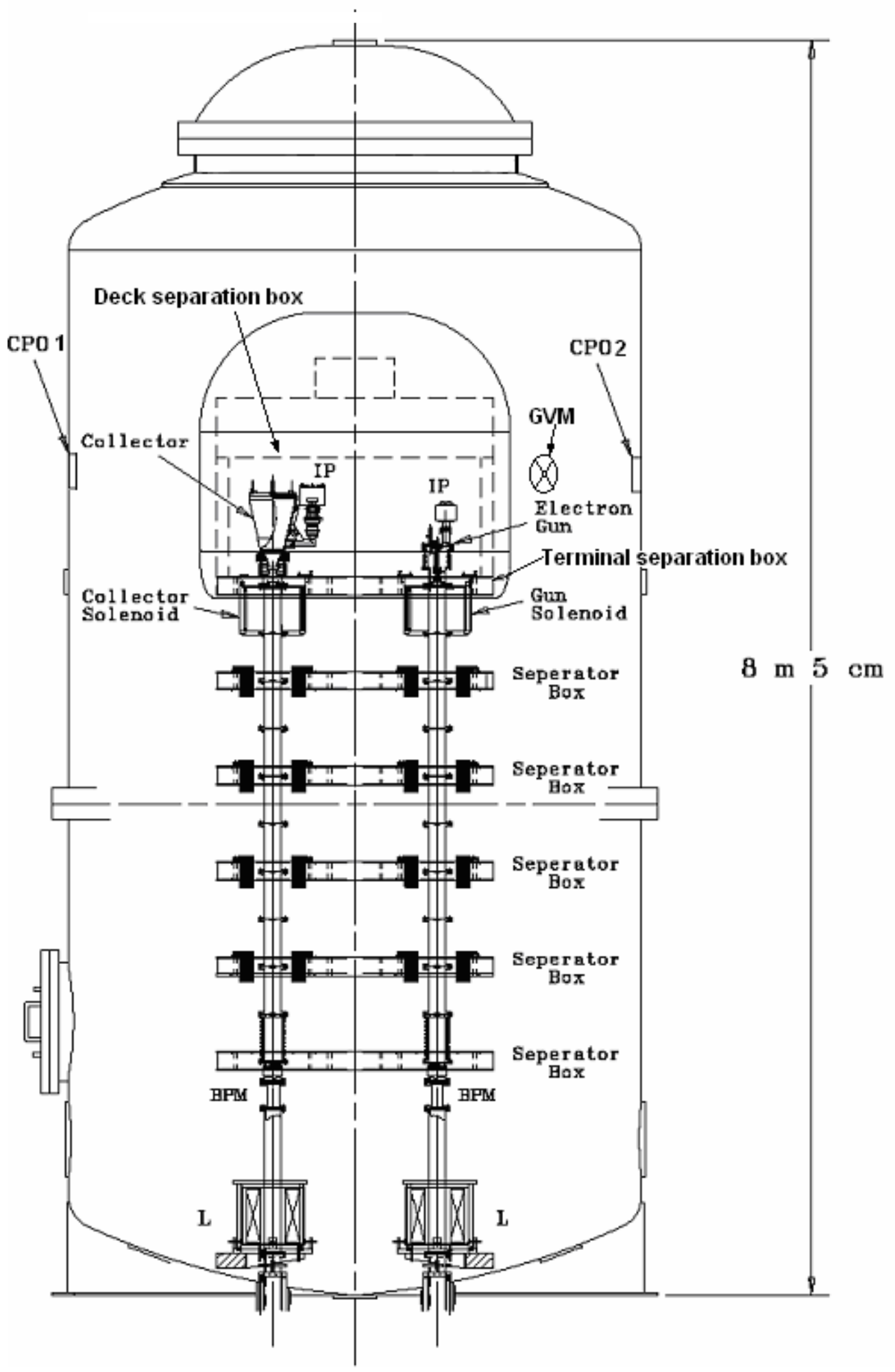

Fig. 2. Mechanical schematic of the Pelletron. CPO indicates capacitive pickups and GVM is a generating voltmeter on the tank wall. IP shows ion pumps. Supports between separation boxes are not shown. 


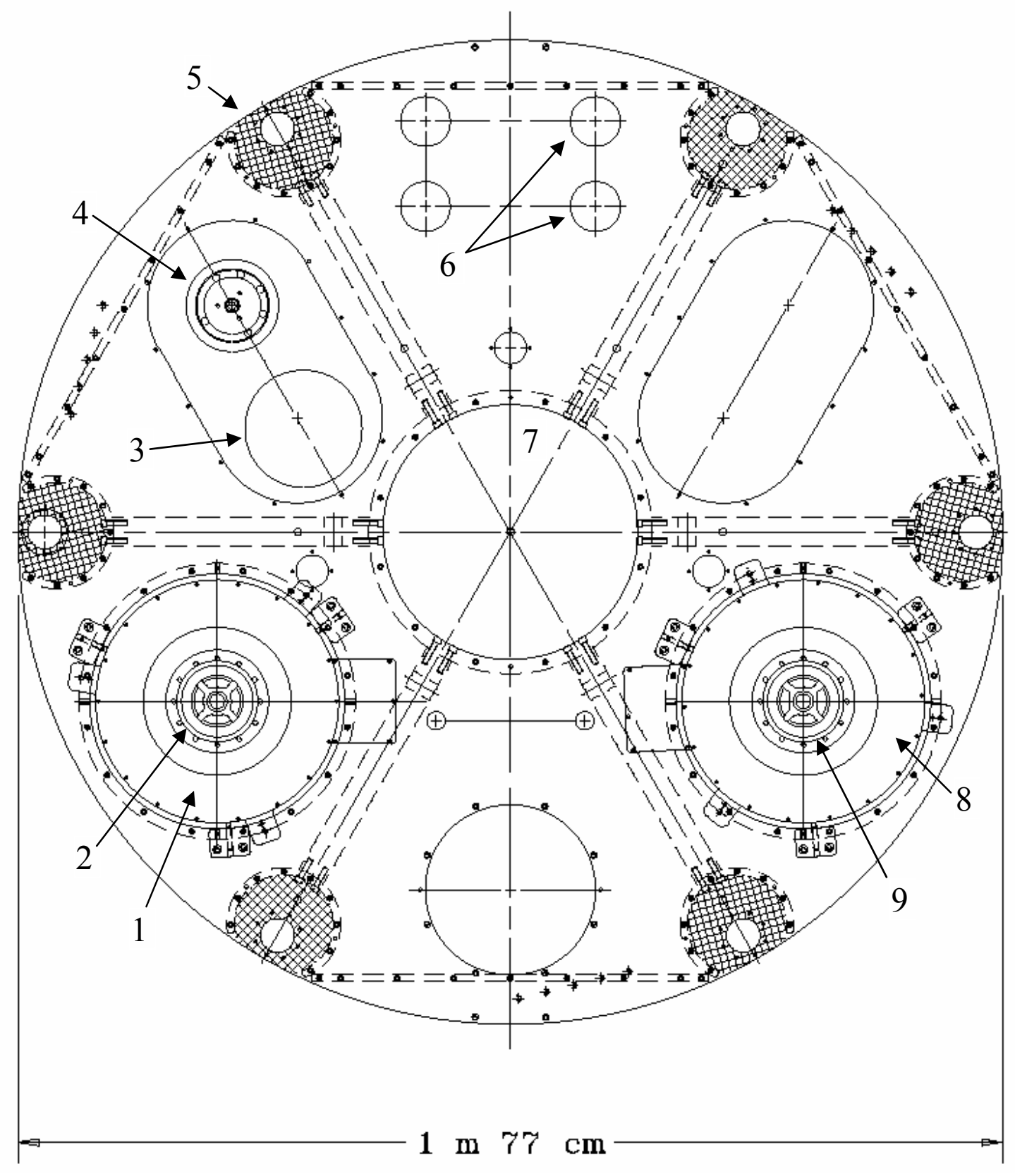

Fig. 3. Cross section of the Pelletron column. 1- solenoid lens, installed at each separation box on the collector side, 2- deceleration tube, 3- generator, 4- shaft, 5- post, 6- chains, 7manhole, 8- solenoid lens, installed at each separation box on the gun side, 9-acceleration tube.

Mechanical vibrations of the Pelletron parts are caused primarily by motion of the Pelletron charging chains and an insulated generator shaft (Fig. 4). The two Pelletron chains consist of aluminum cylinders, pellets, connected by insulating plastic joints. The chains run around sheaves near the tank bottom, where the sheaves are rotated by the chain motor, and at the terminal, where the charge from the pellets couples to the terminal. 


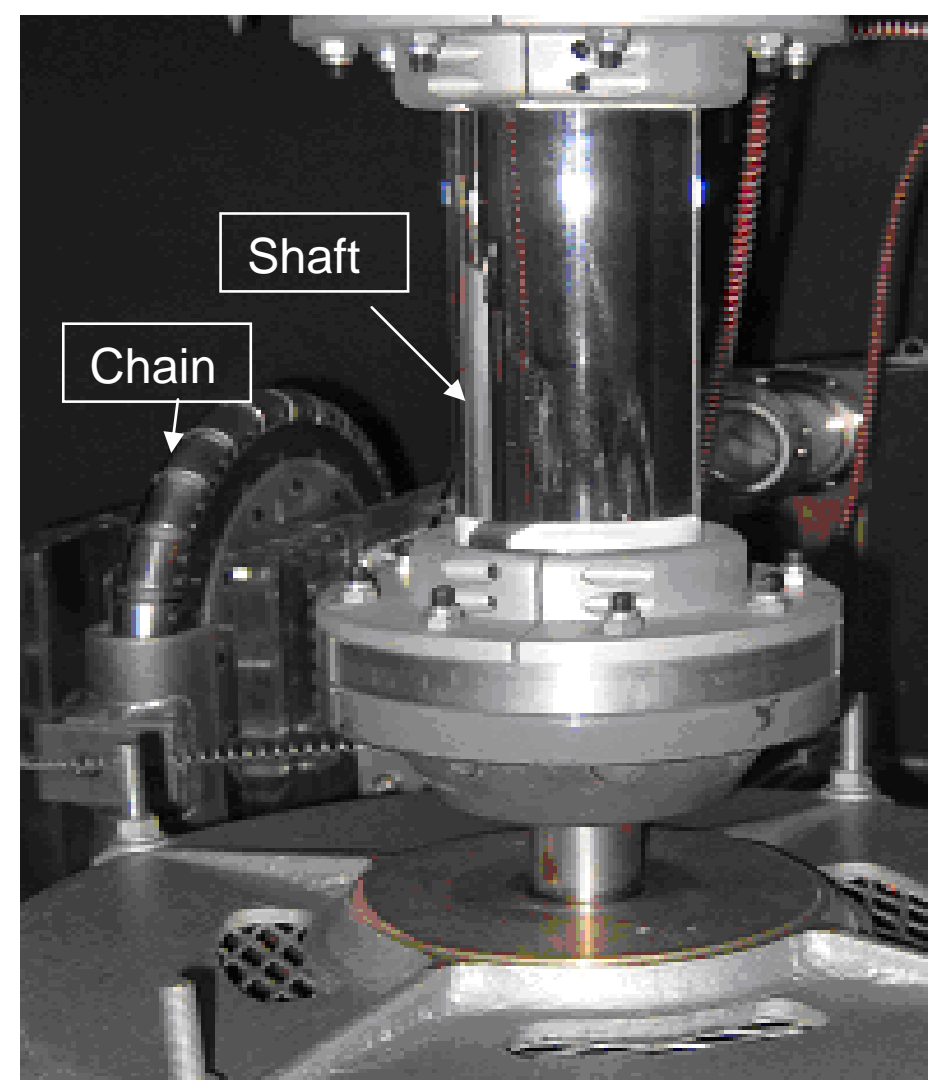

Fig. 4. Photo of the terminal separation box. The chain, shaft, and generator are shown.

The generator shaft is a plastic rod that is used to power equipment in the separation boxes. At the tank bottom, the shaft is rotated by the shaft motor. At each separation box, the shaft is connected by a belt drive to a generator. At the top it drives two generators for the terminal and the HV deck electronics, while also running an SF6 heat exchanger for cooling the collector.

Some characteristics of the shaft and the chain are listed in the Tab. 2.

Table 2: Parameters of the chain and the shaft

\begin{tabular}{|c|c|c|c|}
\hline Parameter & Value & Units & Comment \\
\hline \multicolumn{4}{|l|}{ The chain } \\
\hline $\begin{array}{l}\text { Motor revolution velocity } \\
\text { Motor power } \\
\text { Nominal chain speed } \\
\text { Sheave diameter } \\
\text { Distance between sheave centers }\end{array}$ & $\begin{array}{l}19.3 \\
5.6 \\
18.5 \\
30.5 \\
3.68\end{array}$ & $\begin{array}{l}\mathrm{Rev} / \mathrm{sec} \\
\mathrm{kW} \\
\mathrm{m} / \mathrm{s} \\
\mathrm{cm} \\
\mathrm{m}\end{array}$ & $\begin{array}{l}\text { Nominal; decreases with load } \\
\text { Actual load is } \sim 0.5 \mathrm{~kW}\end{array}$ \\
\hline \multicolumn{4}{|l|}{ The shaft } \\
\hline $\begin{array}{l}\text { Motor revolution velocity } \\
\text { Motor power }\end{array}$ & $\begin{array}{l}29.8 \\
18\end{array}$ & $\begin{array}{l}\mathrm{Rev} / \mathrm{sec} \\
\mathrm{kW}\end{array}$ & \\
\hline
\end{tabular}


Mechanical motion of the column elements may result in two effects: a beam motion and an increase of the energy spread. If the gun or focusing elements move, the beam changes its position in the beam line. The most sensitive motion seems to be at the top of the column, where the gun, the gun lens creating a cathode magnetic field, and the gun solenoid provides initial beam focusing and transport to the acceleration tube (Figs. 5 and 6).

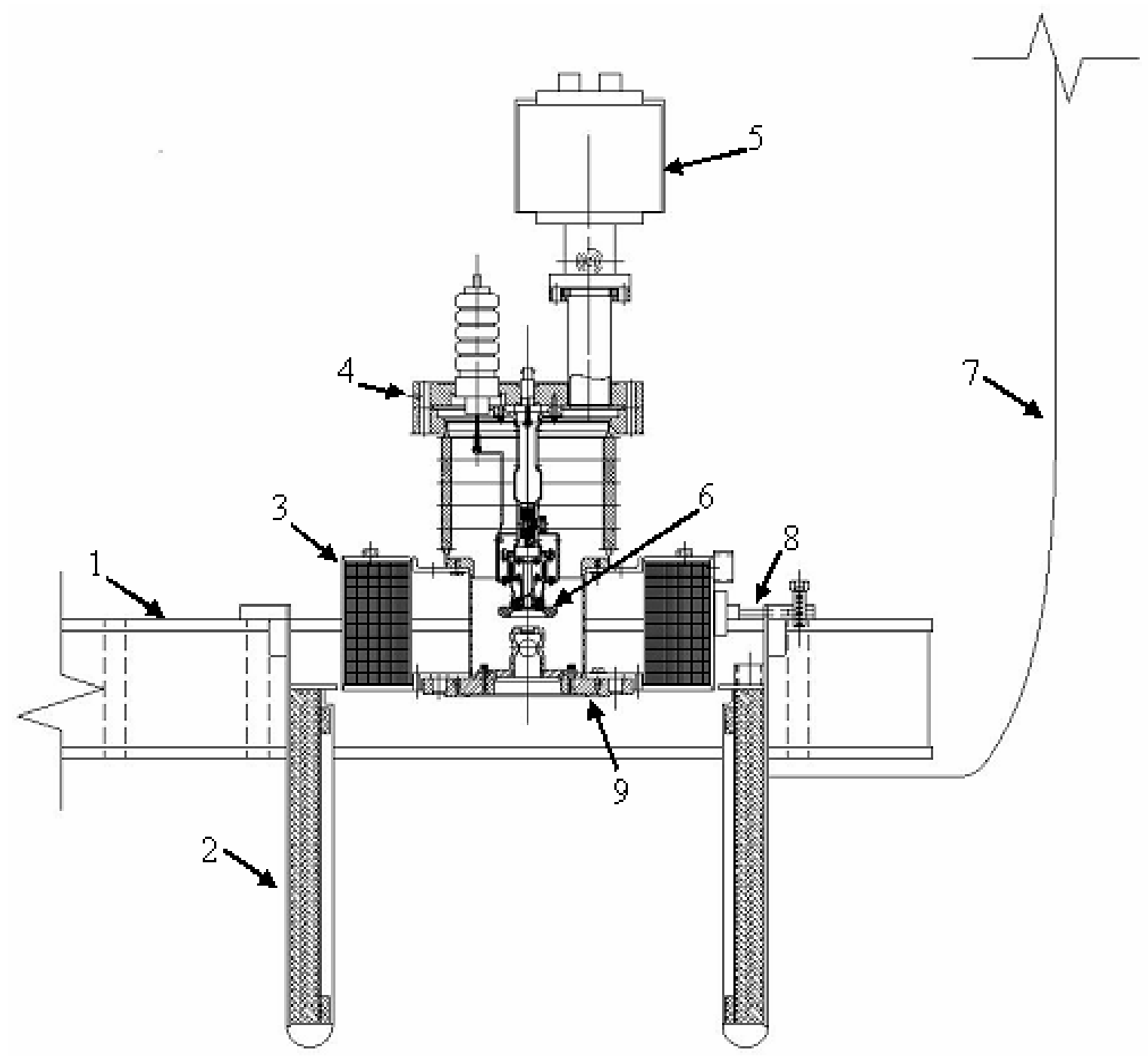

Fig. 5. Electron gun at the terminal. 1- terminal separation box, 2- gun solenoid, 3- gun lens, 4- gun flange, 5- gun ion pump, 6- cathode with a control electrode, 7- terminal shell, 8threaded rods fixing the gun lens and the gun assemble in radial direction, 9- anode flange. In the vertical direction the gun assembly and the gun lens are supported by the acceleration tube, which is not shown here. 


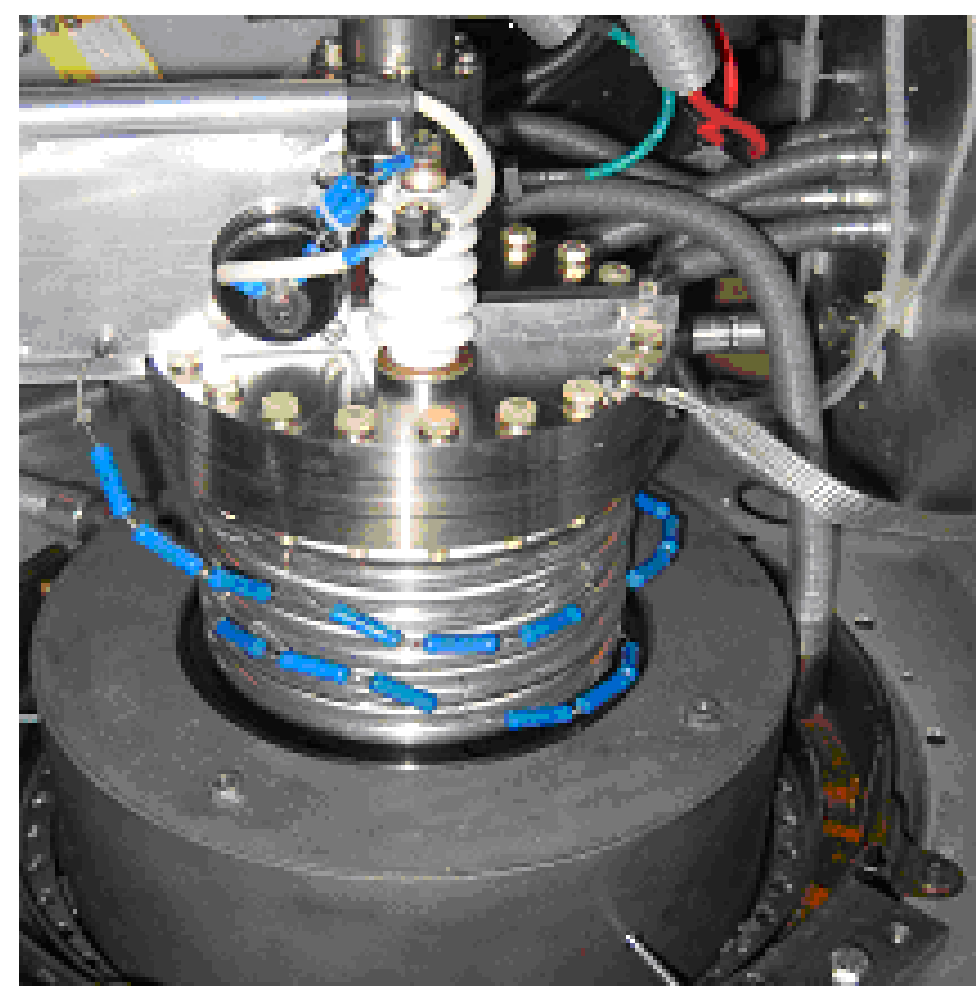

Fig. 6. Photo of elements shown in the drawing of Fig. 5.

The beam energy is determined primarily by the terminal voltage. For a constant charge on the terminal shell, the voltage may change due to a changing capacitance between the terminal and the tank,

$$
\frac{\delta U}{U}=-\frac{\delta C_{t}}{C_{t}}
$$

The change in capacitance from small motions $\delta L$ of the terminal is a second order effect,

$$
\frac{\delta C_{t}}{C_{t}}=\operatorname{coef} \cdot\left(\frac{\delta L}{R_{t}}\right)^{2}
$$

where $R_{t}$ is the radius of the terminal shell. The coefficient is $\sim 1$ and is determined by the geometry. For an estimation, replacing the terminal and the surrounding tank by spheres with corresponding radii, the coefficient is 0.4 . Therefore, even for shifts $\sim 0.1 \mathrm{~mm}$ the resulting relative changes in the terminal voltage are below $10^{-8}$, and the effect is negligibly small.

A noticeable effect of a mechanical motion is on the voltage regulation system. The voltage is stabilized by a feedback system based on signals from a generating voltmeter (GVM) and from two capacitive pickups (or, following NEC's terminology, "pick-outs", labeled CPOs). Their vertical location is shown in Fig. 2. These devices do not measure the voltage but the electric field $E$ on the tank surface. For a simple model of a linear dependence of the field on the distance $L$ between the shell and the tank wall, the CPO signal can be expressed as follows:

$$
\delta U_{c p o}=k \cdot \delta E=k \cdot U \cdot\left(\frac{\delta U}{U}-\frac{\delta L}{L}\right)
$$


where the coefficient $k$ is determined by geometrical factors and by the CPO circuitry. For an individual CPO, $k$ was calibrated to be $2 \cdot 10^{-3}$. The tolerable level of potential variations is, $\delta U / U \sim 1 \cdot 10^{-4}$, thus for $L=890 \mathrm{~mm}, \delta L \sim 0.09 \mathrm{~mm}$. Therefore, a mechanical motion of the terminal shell with amplitude of tens of microns may contribute to the energy stability.

Effects mentioned above will be discussed in the following sections.

\section{Diagnostics}

This paper discusses measurements of three different groups of parameters that may be interconnected by mechanical motion of the Pelletron: mechanical vibrations measured by mechanical sensors with the Pelletron tank open, CPO signals, and beam oscillations. The mechanical vibration data were taken for several combinations of the shaft and chain motors being off or on. Displacements of the elements were measured by three types of sensors.

\subsection{Direct diagnostics of displacements of the Pelletron elements}

Rough measurements caused by the revolving shaft were done with a piezo vibration meter, Columbia Research Lab - VM-103, operating in the "displacement" mode. The meter measures an rms value of a displacement in the bandwidth of 8-1200 $\mathrm{Hz}$.

\subsection{Measurements of the Pelletron vibrations with velocimeters}

Vibrations of the upper separation box of the Pelletron, caused by the revolving shaft, were studied with two velocimeters of SM-3KV type. Sensors of these devices are mechanical pendulums sensitive to either horizontal or vertical oscillations. Both velocimeters were installed on the upper separation box (terminal). The velocimeters use electronic feedback to linearize and approximately equalize the sensitivities throughout the bandwidth of $0.05-100 \mathrm{~Hz}$. The pendulum's free oscillation period is $\sim 1-3 \mathrm{sec}$. Velocimeters sensitivity with an additional attenuation of 10 is shown in Fig.7.

The velocimeters require a large flat surface to be installed, and in the case of Pelletron vibration, their high sensitivity leads to saturation of the amplifiers. To avoid the saturation we were forced to decrease the bandwidth of the amplifiers using the frequency cutoff by the measurements. This limited the use of the velocimeters to a qualitative verification of geophone measurements.

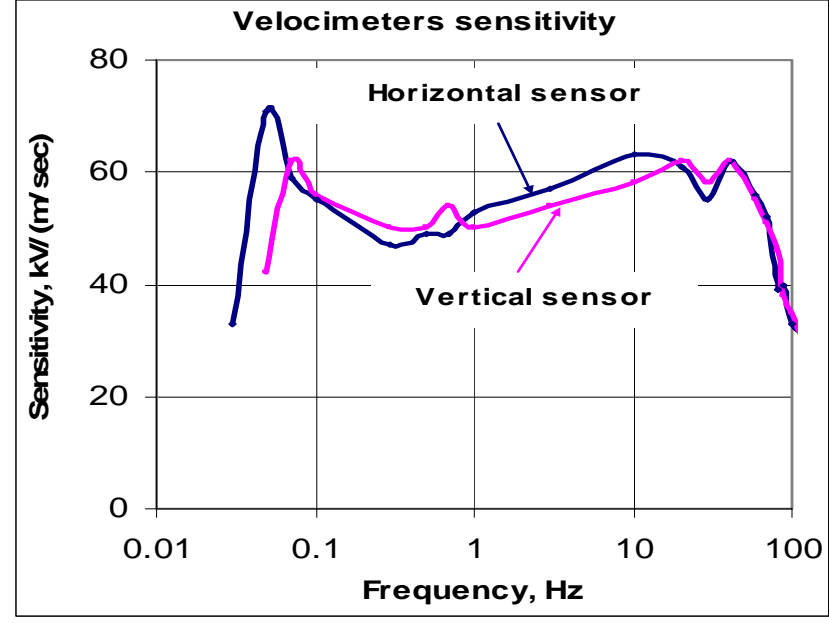

Fig. 7. Calibration curves of the velocimeters 


\subsection{Measurements of the Pelletron vibrations with geophones}

The major portion of the Pelletron vibration measurements were done with a compact module containing three geophones, Geo Space HS-1 (Figs. 8 and 9). The eigenfrequency of the geophones is $4.5 \mathrm{~Hz}$, and the average sensitivity is $1.15 \mathrm{~V} / \mathrm{in} / \mathrm{s}$. The corresponding calibration curve is presented in Fig. 10, curve A.

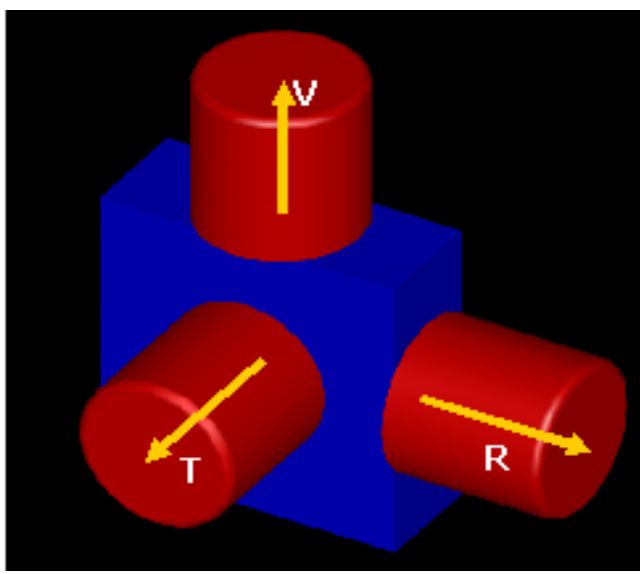

Fig. 8. Layout of the measuring module (R-radial, T-tangential, V-vertical HS-1 sensors, respectively)

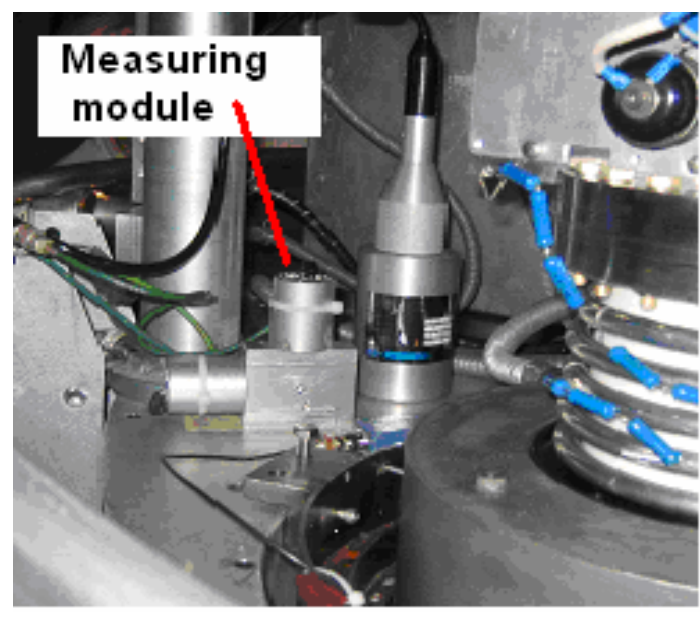

Fig. 9. Geophone HS-1 measuring module on the upper separation box.

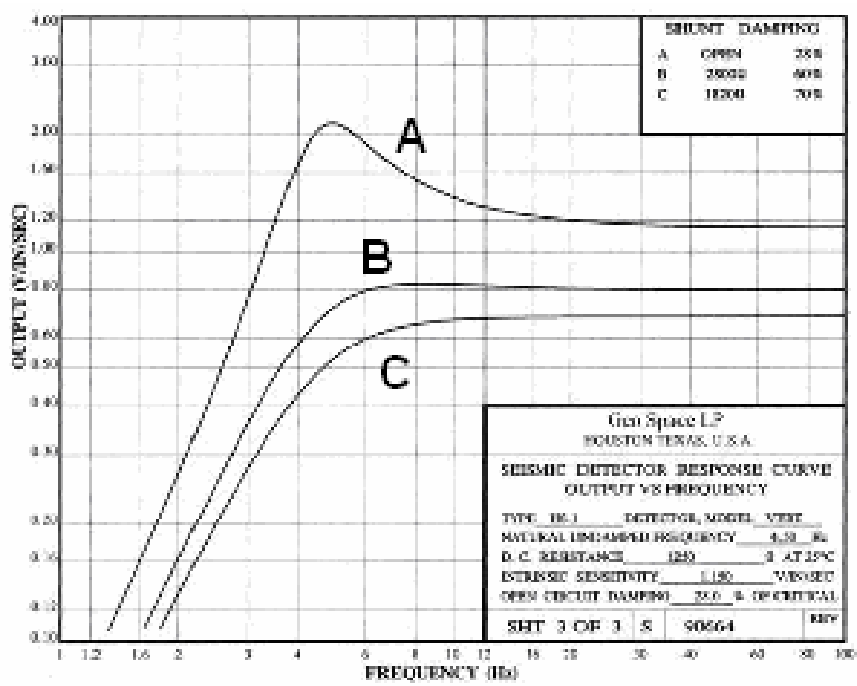

Fig. 10. Calibration curves of HS-1 geophone.

\subsection{Terminal shell vibration and CPOs}

Two capacitive pickups (called CPO-1 and CPO-2) are mounted on exactly opposite sides of the Pelletron tank to measure the electric field variations in the bandwidth up to $1 \mathrm{kHz}$. The signals can be displayed separately or after a hardware summing. If the terminal shell doesn't move but the terminal voltage changes, both CPOs show identical signals with the same phase. On the other hand, if the voltage is constant, phases of the CPO signals are determined by oscillation modes of the terminal shell. For 
a particular case, motion of the terminal shell along the line connecting the CPO axis results in a signal of the same amplitude but opposite phase. Analysis of the CPO signals may provide information about the terminal motion.

\subsection{Beam position monitors}

Analysis of the beam motion was performed with a system of capacitive pickups, referred to as BPMs (beam position monitors). The beam transport line included 19 pairs of BPM detectors. The measurements under discussion were made with a DC beam of 20-200 mA, which was slightly intensity modulated in the gun at a frequency of $32 \mathrm{kHz}$.

A BPM detector consists of two $5 \mathrm{~cm}$ split plate assemblies with an inner diameter of 2 inches. The four detector plates are connected with short lengths of coax to a four-channel low-noise charge preamplifier. The output of the preamplifier is connected to a digital signal receiver (DSR) VXI module, Fig. 11.

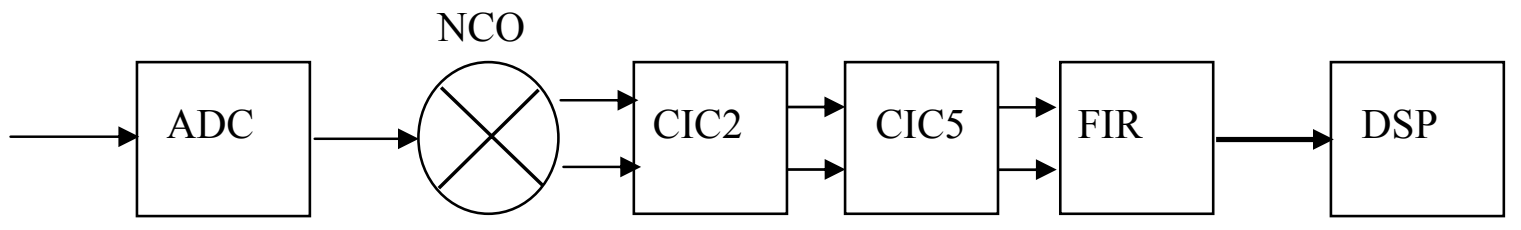

Fig. 11. Block diagram of 1 channel of the DSR module used in the electron cooling BPM system. The pre-amplifier signal enters from the left. It is digitized by the ADC and demodulated by the NCO (numerically controlled oscillator). The resulting I/Q data is passed sequentially through a $2^{\text {nd }}$ order and $5^{\text {th }}$ order cascaded integrating comb filter (CIC) and a 256 tap FIR filter. A DSP processor reads this filtered data and calculates the beam position.

The DSR samples the BPM signal at $65 \mathrm{MHz}$ and is de-modulated at $32 \mathrm{kHz}$ to remove the small modulation frequency added to the electron beam to enable detection by the DSR. The signal is then passed through three hardware filters, two cascaded integrating comb filters, and a 256 tap FIR filter. These filters decimate the signal and reduce its bandwidth to $800 \mathrm{~Hz}$. An on-board DSP processor (Analog Devices SHARCTM $\mathrm{AD}-21062$ ) reads this data at a rate of $21.6 \mathrm{kHz}$.

Software running on the SHARC calculates the signal intensity for each plate and applies a difference over sum method for calculating the beam position.

$$
x=S \frac{A-k B}{A+k B}+O,
$$

where $A$ and $B$ are the plate intensities calculated from the square root of the sum of the squares of the I/Q signals for that plate. $S$ and $O$ are scale and offset terms determined by the physical construction of the BPM, and $k$ is a calibration factor which accounts for small differences in signal path gain between $A$ and $B$ plates. The value of $k$ is obtained from a calibration procedure which involves passing a known signal with a fixed $A / B$ ratio through the pre-amplifier and DSR. The measured plate intensities are then used along with the known ratio to calculate a calibration constant for each BPM channel.

To detect oscillations at the Pelletron the BPM system was configured to calculate positions at a rate of $296.804 \mathrm{~Hz}$. The system acquired 1024 data points for all installed BPM detectors and placed the data in a buffer. The buffered data was then extracted and 
stored for offline FFT analysis. This allows detection of frequencies up to $\sim 150 \mathrm{~Hz}$ with the resolution $0.3 \mathrm{~Hz}$.

\section{Beam motion analysis}

Analysis of data taken simultaneously at all BPMs allows calculation of the amplitudes, frequencies, and, to some extend, locations of the source of beam motions. Comparison of oscillations in low and high dispersion regions gives an estimation of the energy fluctuations. Measurements were done at an accelerating voltage of 3.5 MV and a DC beam current of $100 \mathrm{~mA}$. The magnetic field in the cooling section solenoid was 75 Gauss. Neighboring BPMs in the cooling section were separated by $2036 \mathrm{~mm}$.

\subsection{Beam oscillation in the cooling section}

BPM data, comprised of 1024 points at the sampling frequency of $\sim 297 \mathrm{~Hz}$, were taken along the whole line. The time domain signals are dominated by the BPM noise, Fig. 12. The respective FFT transformation of this signal is presented in Fig. 13.

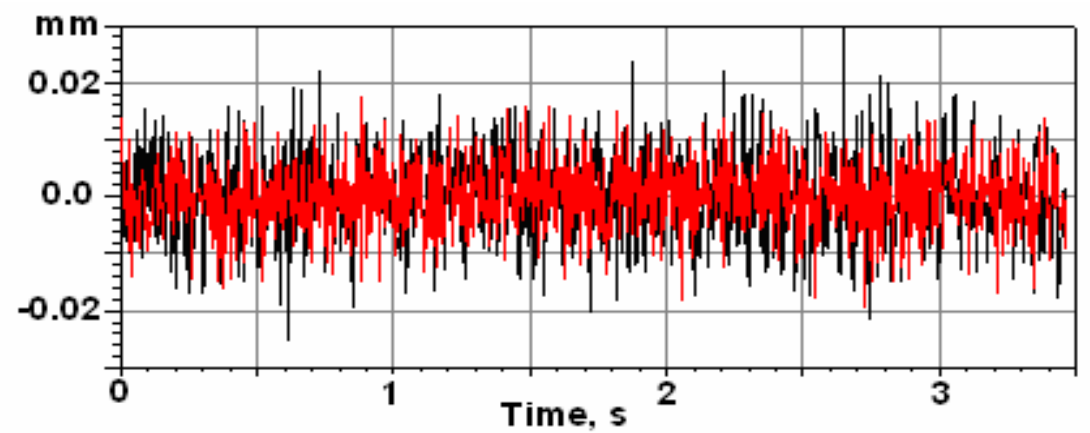

Fig. 12. Deviations of the beam centroid in $\mathrm{X}$ (black) and $\mathrm{Y}$ (red) directions in $\mathrm{mm}$ measured in the supply section during the sampling.

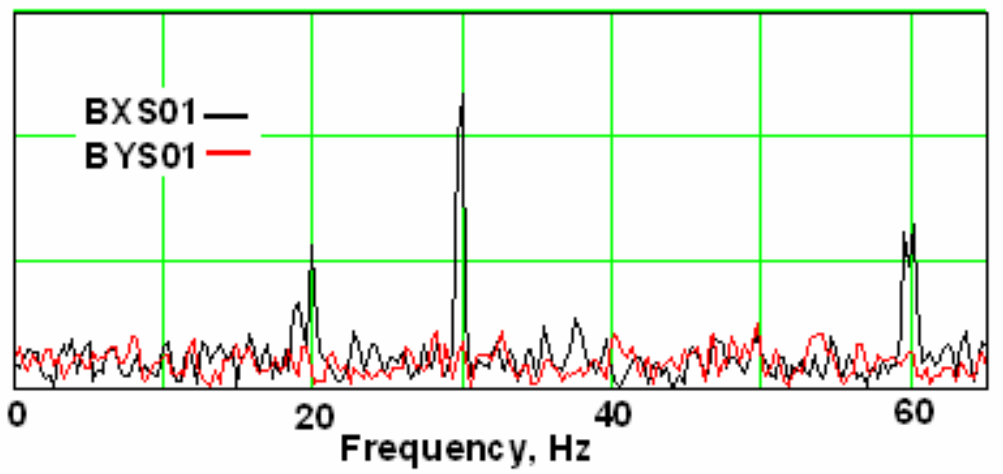

Fig. 13. FFT transformation of signals from BXS01 and BYS01 BPMs.

Distinguishing between beam-related and unrelated signals can be obtained from the spectra observed in the cooling section. Here the beam trajectory is given by a helix:

$$
\begin{aligned}
& x(s)=\left[x(0)-y^{\prime}(0) / k\right]+x^{\prime}(0) \sin (k s) / k+y^{\prime}(0) \cos (k s) / k \\
& y(s)=\left[y(0)+x^{\prime}(0) / k\right]+y^{\prime}(0) \sin (k s) / k-x^{\prime}(0) \cos (k s) / k
\end{aligned}
$$


For any frequency, only 4 unknown values at the entrance of the cooling section determine 16 values at 8 BPMs. Thus, beam-driven signals at the BPMs must have a strong correlation with each other, assuming that all the sources of this beam motion are upstream of the cooling section. Observing spectra presented in Fig. 14 shows that only a few significant peaks appear at almost all BPMs. The most pronounced peaks are at 29.8 and $\sim 60 \mathrm{~Hz}$. More accurately, the second peak consists of two close, but independent peaks, located at 59.5 and $60 \mathrm{~Hz}$, Fig. 13. It is natural to suppose that the $59.5 \mathrm{~Hz}$ peak is a second harmonic of the $29.8 \mathrm{~Hz}$ oscillation, while the $60 \mathrm{~Hz}$ peak is a frequency of the power circuit.

All three peaks were fitted for consistency with the helix trajectory (4.1), and the errors were found smaller then $10 \%$ for all cases. This result shows that all 3 peaks relate to the beam motion. Together, they give $\sim 40-60 \mu \mathrm{m}$ of the rms offset in the cooling section.

The spectra have also a $20 \mathrm{~Hz}$ peak that can be related to the chain transverse oscillations (see Section 5). However, the peak amplitudes are considerably lower than the main ones, and the noise doesn't allow for a detailed analysis.
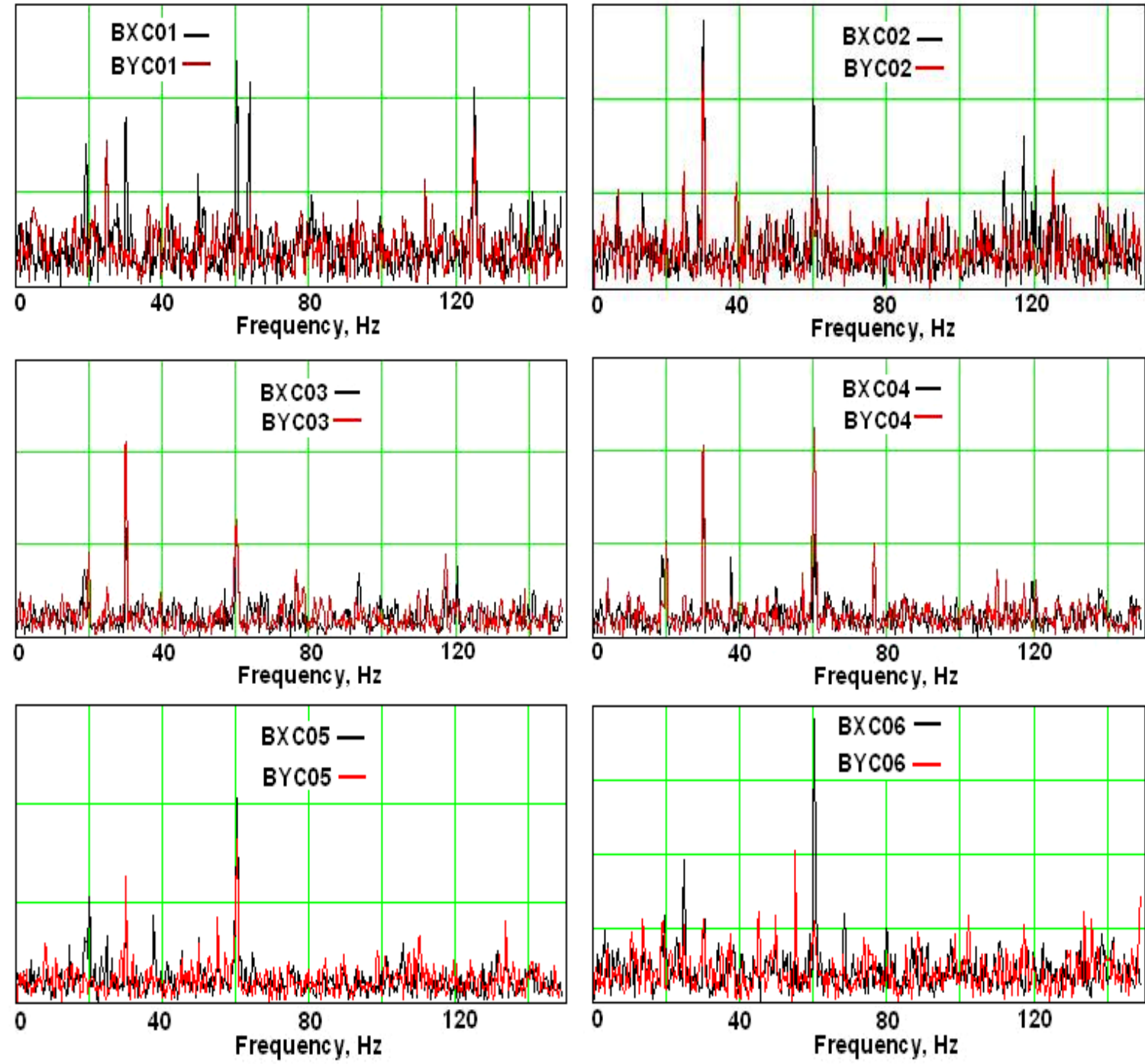

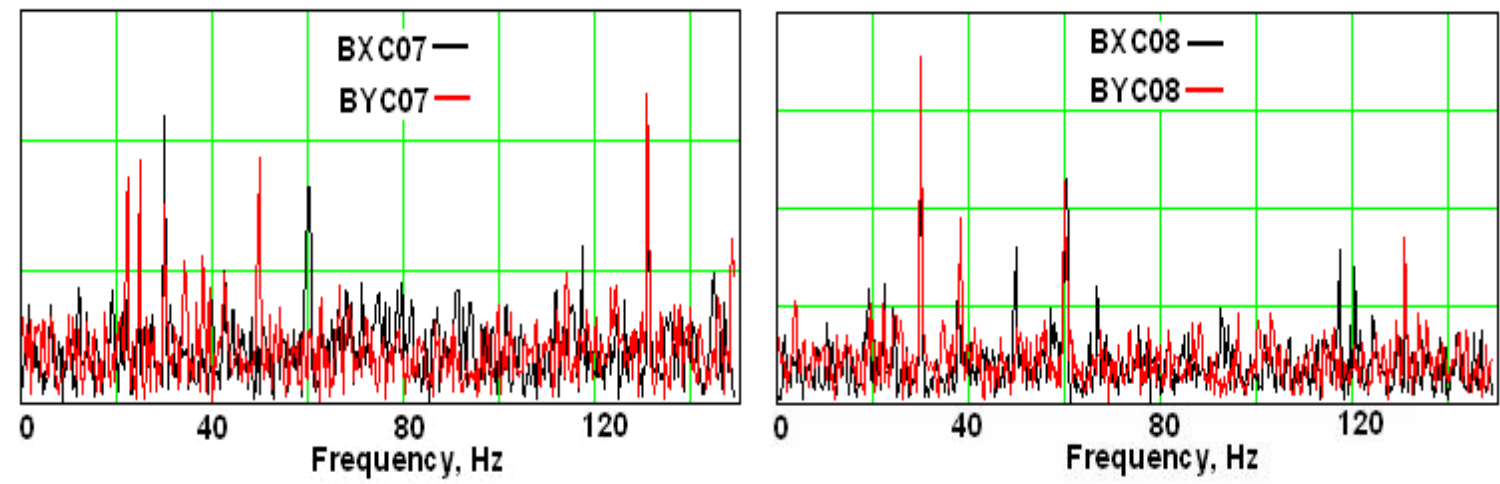

Fig. 14: FFT transformations of signals from BPMs along the cooling section.

These three frequencies can be extracted from the initial row BPM signals, and the remaining signals could be checked on mutual correlations. The result is that the correlation coefficients of these remaining signals cannot be distinguished from their uncorrelated values. Thus, the beam-related signals are dominated by and mostly limited to the three peaks.

\subsection{Location of oscillation sources}

Some information can be extracted about sources of these oscillations. Namely, for every peak, the initial conditions at the entrance of the cooling section can be extracted. After that, these initial conditions can be back propagated along the optical structure, and the calculated results compared with the BPM data at these frequencies for the upstream BPMs. Significant deviations of the calculated and measured numbers, if seen at some BPM, would signal a source of the perturbation between this and the nearest downstream BPM. For any beam-related signal, the real and imaginary parts of the FFT can be analyzed separately. The results for 29.8, 59.5 and $60 \mathrm{~Hz}$ peaks are shown in Figs. 15a-f. For 29.8 and $59.5 \mathrm{~Hz}$, all the measured points are seen to be in reasonable agreement with the simulations. Thus, the source of the beam motion for these frequencies is located somewhere upstream of BS1 BPM ( $3^{\text {rd }}$ from the left). For $60 \mathrm{~Hz}$, the discrepancy between the measurements and calculations is significant. So, for this frequency, there is at least one more source of the perturbation between the cooling section and BS3 BPM.

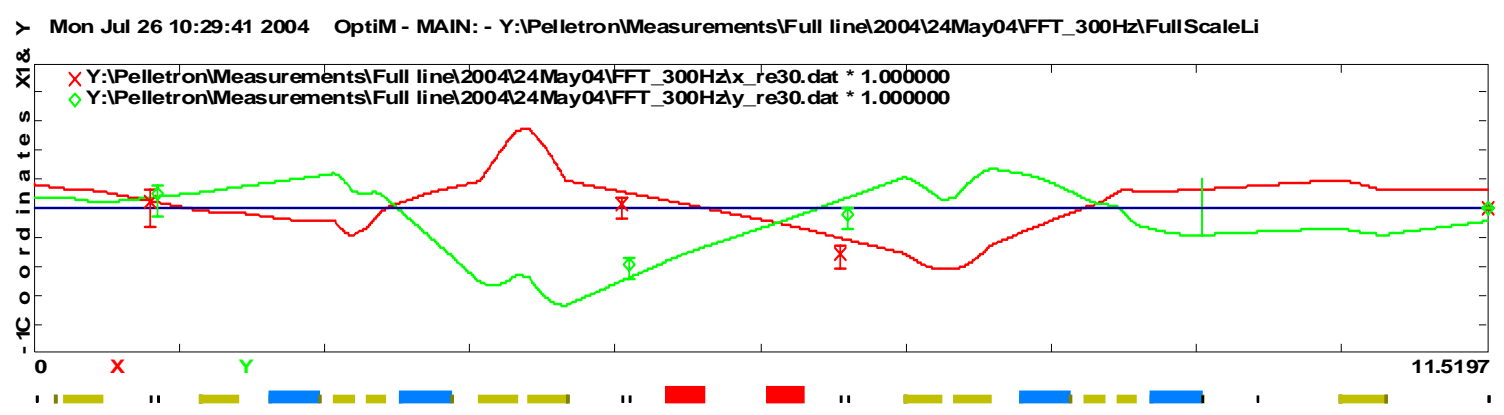

Fig. 15a: Reverse propagation of the initial conditions from the cooler entrance back to the Pelletron, FFT for $29.8 \mathrm{~Hz}$, real part. Points with error bars relate to measurements (the 
BPMs, from left to right: BS3, BS2, BS1); lines show the calculations. Red and green colors are for the horizontal and vertical transverse directions.

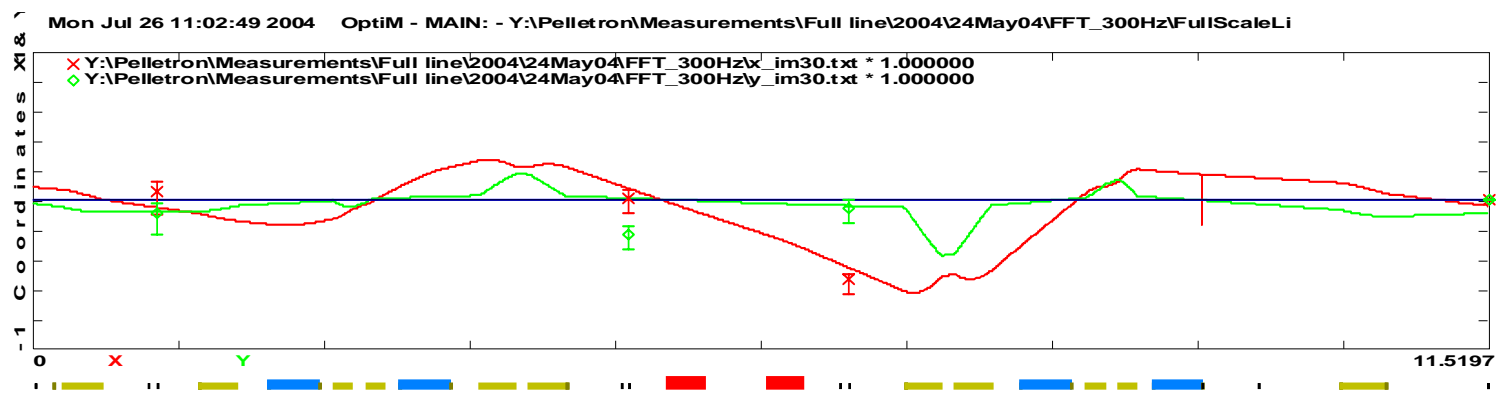

Fig. 15b: The same as above, $29.8 \mathrm{~Hz}$, imaginary part.

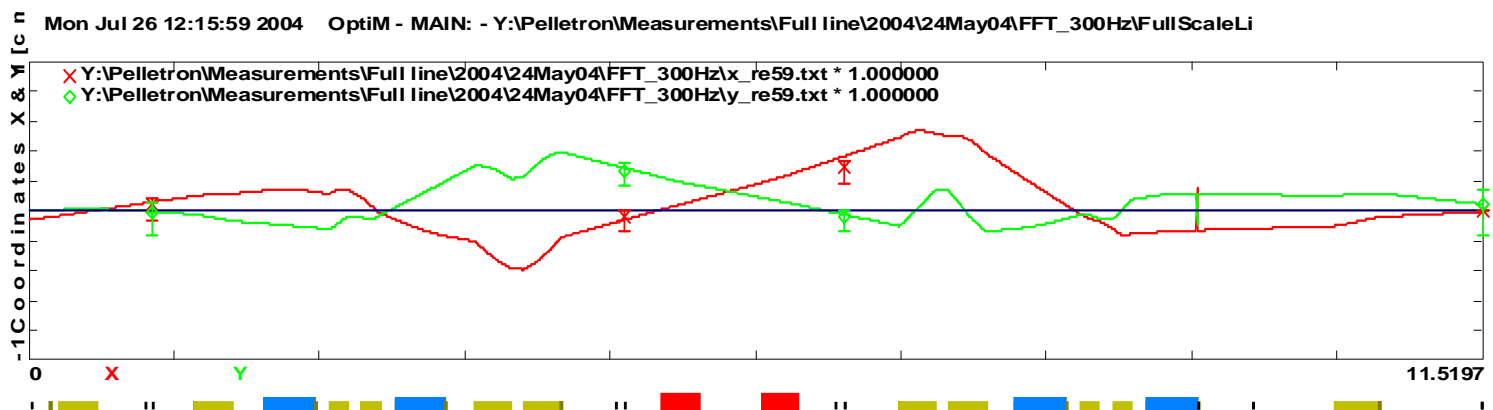

Fig. 15c: $59.5 \mathrm{~Hz}$, real part

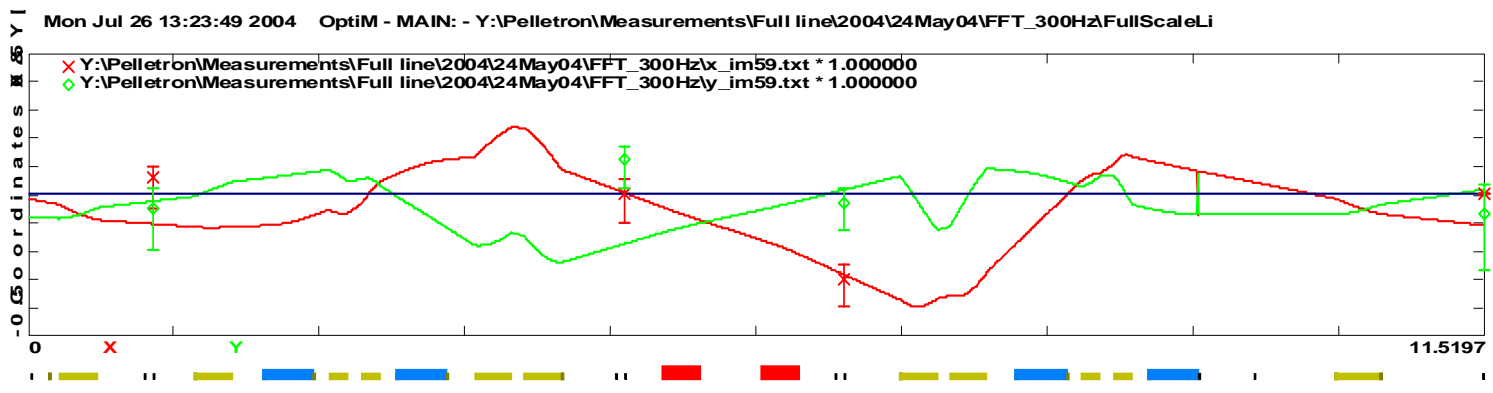

Fig. 15d: $59.5 \mathrm{~Hz}$, imaginary part

Mon Aug 02 11:25:54 2004 OptiM - MAIN: - Y:IPelletron IMeasurementsIFull linel2004|24MayO4IFFT 300HzIFullScaleLi

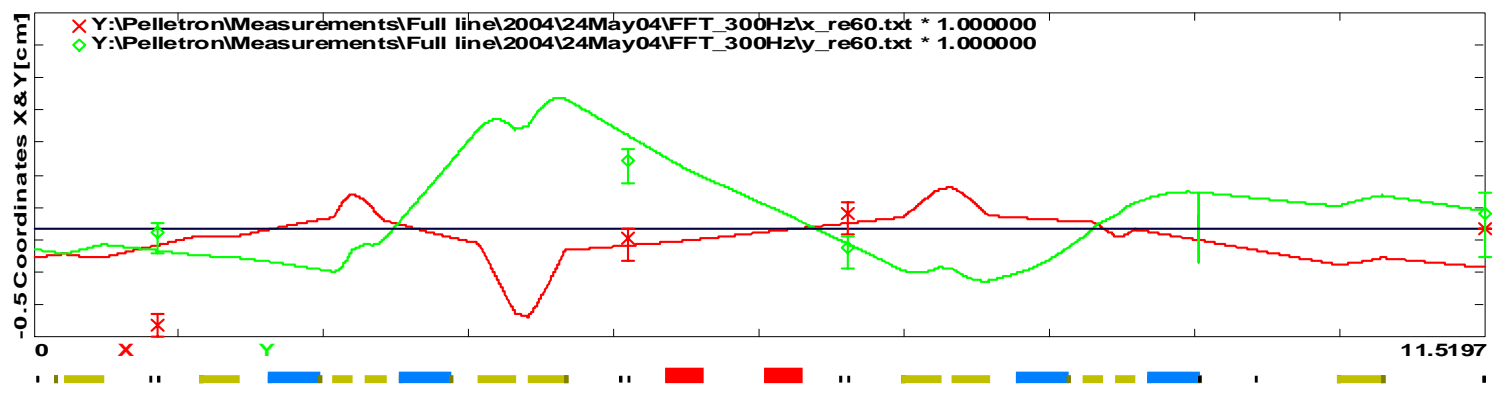

Fig. 15e: $60 \mathrm{~Hz}$, real part 
Mon Aug 02 11:26:58 2004 OptiM - MAIN: - Y:IPelletronIMeasurementsIFull linel2004|24May04IFFT_300HzIFulIScaleLi

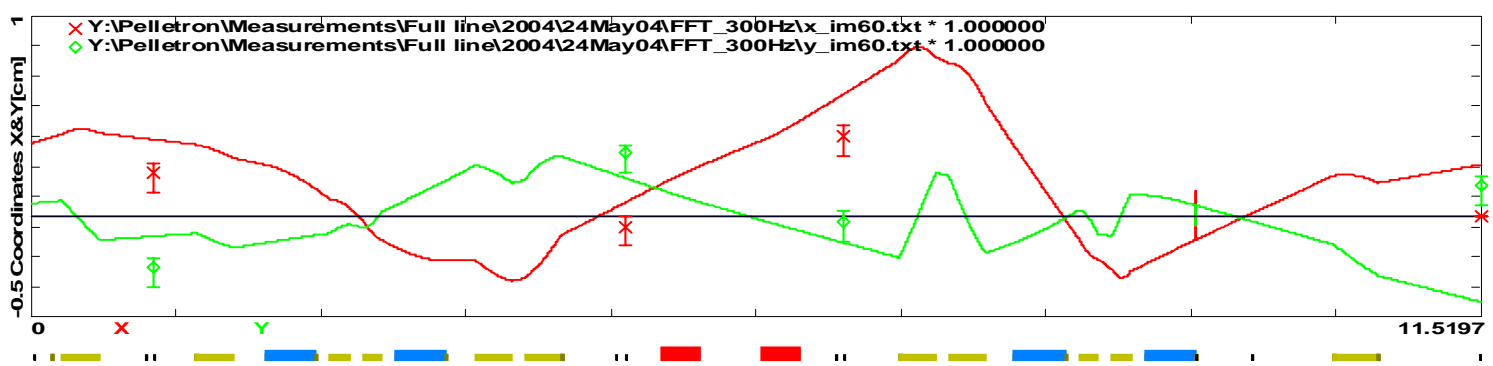

Fig. 15f: $60 \mathrm{~Hz}$, imaginary part

Altogether, these beam oscillations give $\sim 20 \mu \mathrm{rad}$ of rms angle in the cooling section, which is $\sim 10$ times smaller that the total tolerable angle.

\subsection{Energy fluctuation}

Direct propagation of the restored initial conditions at the cooler entrance can be done as well. The results for 29.8, 59.5 and $60 \mathrm{~Hz}$ are shown in Figs. 16 a-f.

Tue Dec 14 11:58:41 2004 OptiM - MAIN: - Y:IPelletronIMeasurementsIFull linel2004|24May04IFFT_300HzIFulIScaleLi

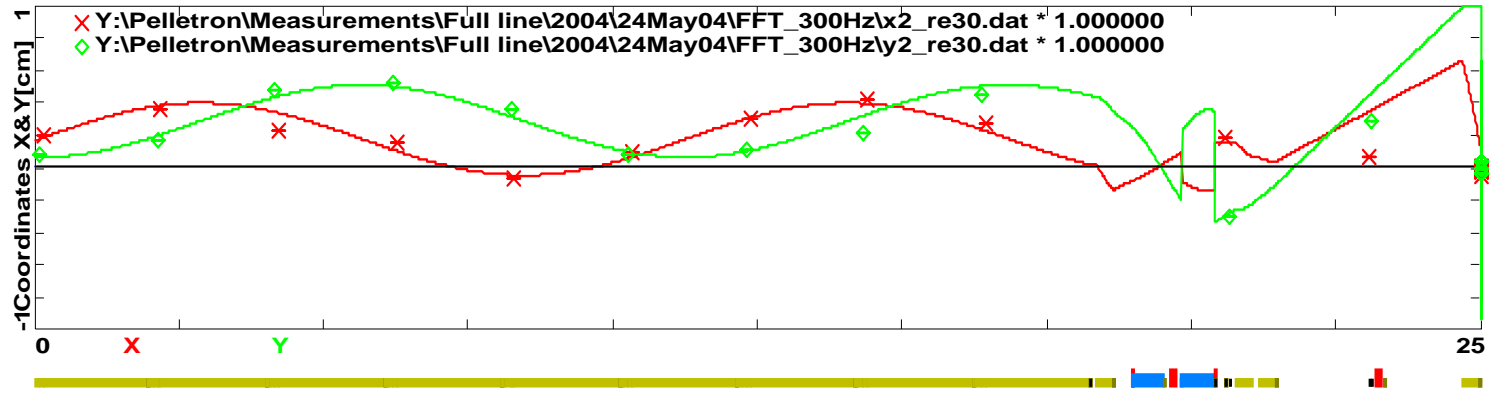

Fig. 16a. Forward propagation of the calculated initial conditions for FFT at $29.8 \mathrm{~Hz}$, real part.

Tue Dec 14 12:00:23 2004 OptiM - MAIN: - Y:IPelletronIMeasurementsIFull linel2004I24May04IFFT_300HzIFulIScaleLi

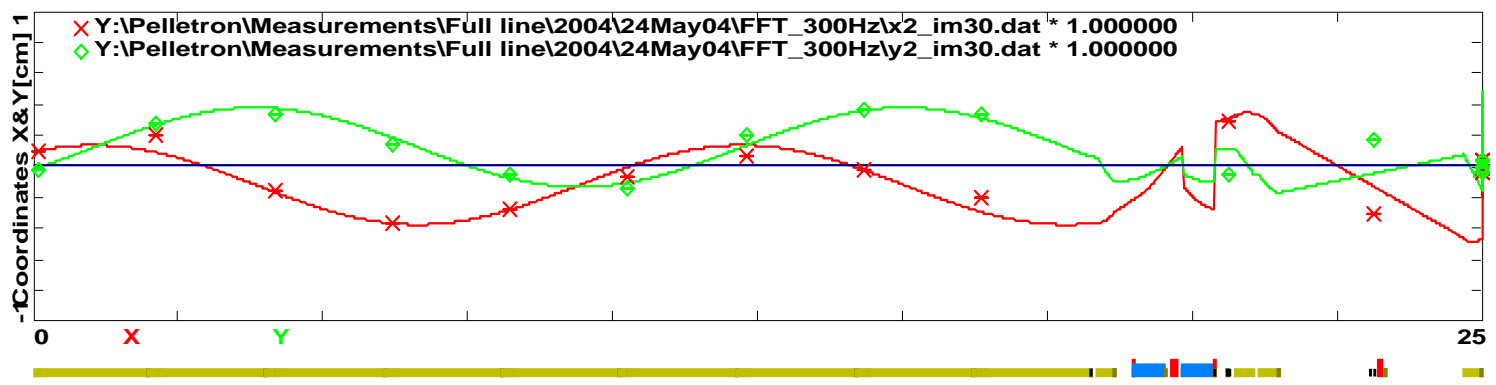

Fig. 16b: The same as fig. 16a, $29.8 \mathrm{~Hz}$, imaginary part. 
Tue Dec 14 13:30:34 2004 OptiM - MAIN: - Y:IPelletronIMeasurementsIFull linel2004I24May04IFFT_300HzIFulIScaleLi

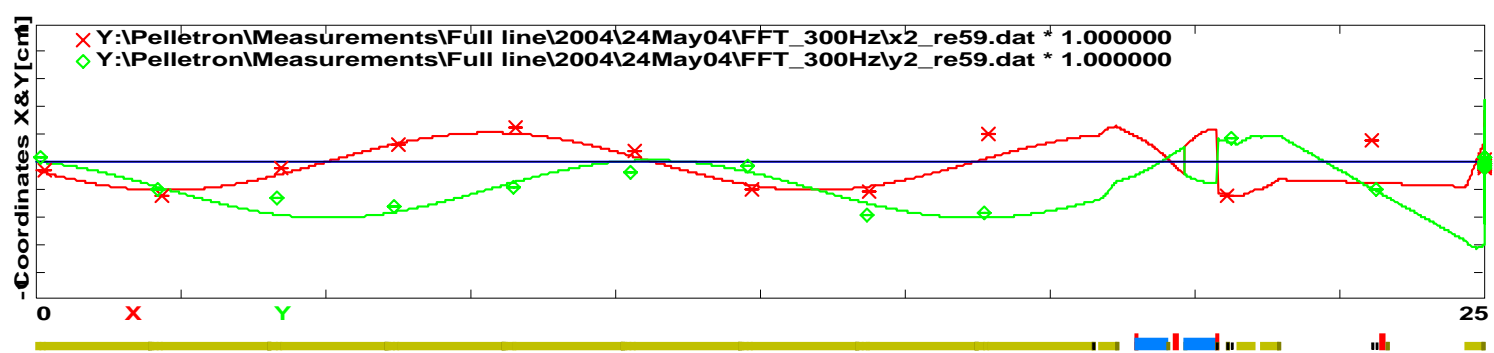

Fig. 16c: $59.5 \mathrm{~Hz}$, real part.

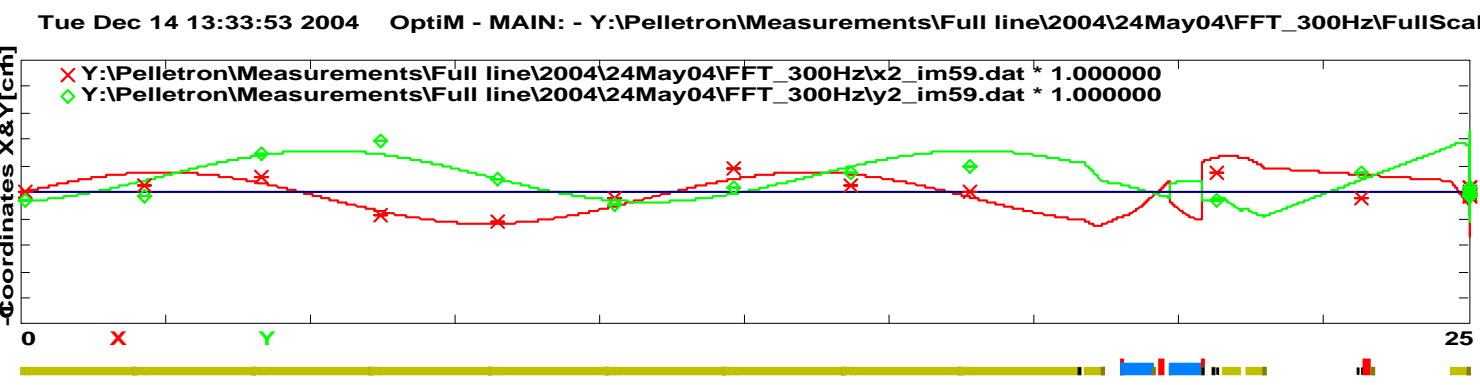

Fig. 16d: $59.5 \mathrm{~Hz}$, imaginary part.

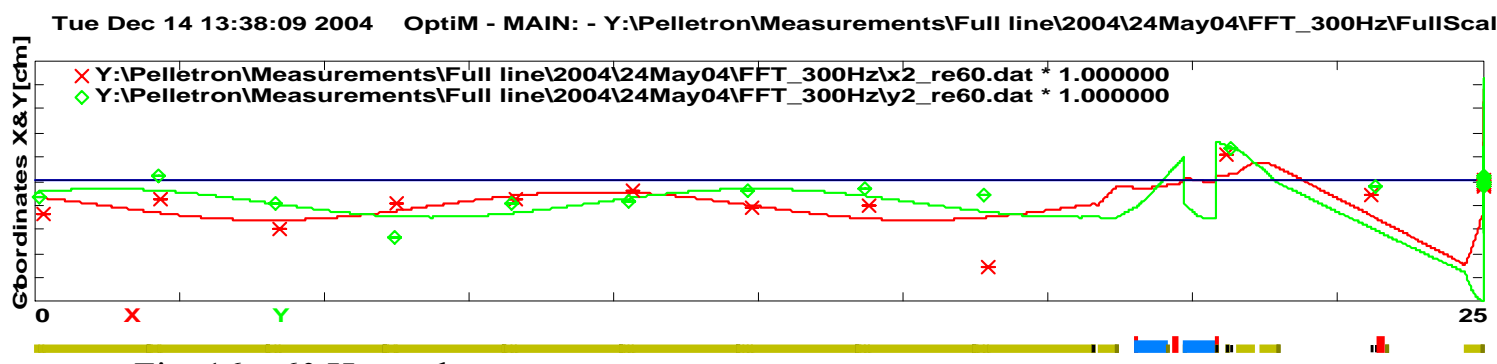

Fig. 16e: $60 \mathrm{~Hz}$, real part.

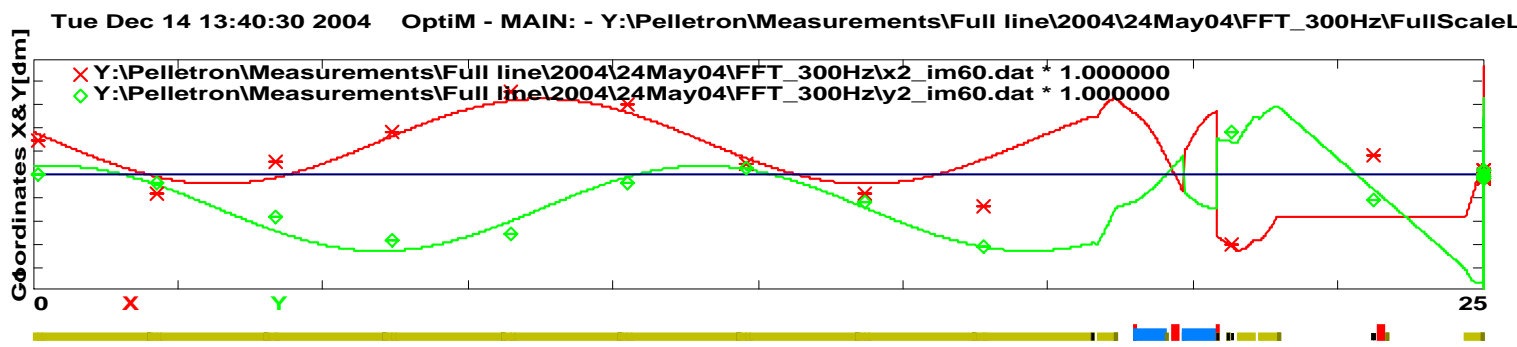

Fig. 16f: $60 \mathrm{~Hz}$, imaginary part.

Several features deserve to be mentioned:

First, the agreement inside the cooling section and at the first BPM after that $(\mathrm{Bx} / \mathrm{yR} 1)$ is rather good. For this optics, there is no dispersion upstream of the $180^{\circ}$-bend (marked with blue rectangles by OptiM). BPM ByR1 is located just after the bend, where the vertical dispersion is $130 \mathrm{~cm}$.

Second, for all the plots of Fig. 16, there is only one where the vertical (green) point of ByR1 deviates from the trajectory more than the error bar; namely, it is Fig. 16b, related to the imaginary part of the FFT for $29.8 \mathrm{~Hz}$. This deviation sets an upper limit for 
the energy oscillation. A plot in Fig. 17 shows a time-domain signal for ByR1 at a frequency band of $29.8 \pm 0.4 \mathrm{~Hz}$. As is seen here, the maximal amplitude is $\sim 20$ micron.

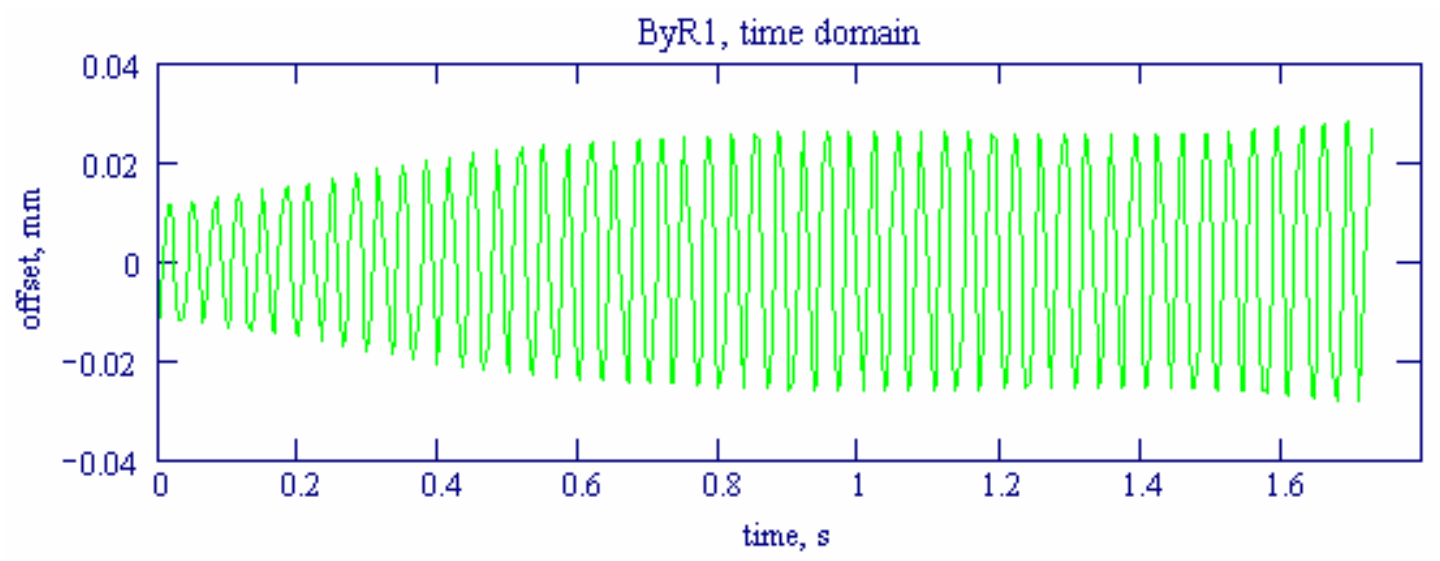

Fig. 17: Offset at ByR1 at a frequency band of $29.8 \pm 0.4 \mathrm{~Hz}$.

The bandwidth here is determined by the resolution of the FFT; however, the maximum amplitude is almost independent of the bandwidth. The discrepancy of ByR1 at Fig. 16b is about the signal value itself, $\sim 20$ micron. It sets the limit on the relative energy oscillation

or:

$$
\begin{aligned}
\Delta p / p & \leq 2 \cdot 10^{-5}, \\
\Delta E & \leq 100 \mathrm{~V} .
\end{aligned}
$$

One more noticeable feature of Figs. 16 is that the measurement from the last shown $\mathrm{BPM}, \mathrm{Bx} / \mathrm{yR} 2$ usually deviates significantly from the calculated trajectory. This discrepancy may mean that there are additional sources of perturbations between BR1 and BR2

Figs. 18a, and 18b, shows 3-5 Hz band-pass filtered ByR01 signal and FFT spectrum of the signal respectively, confirming estimations (4.2).

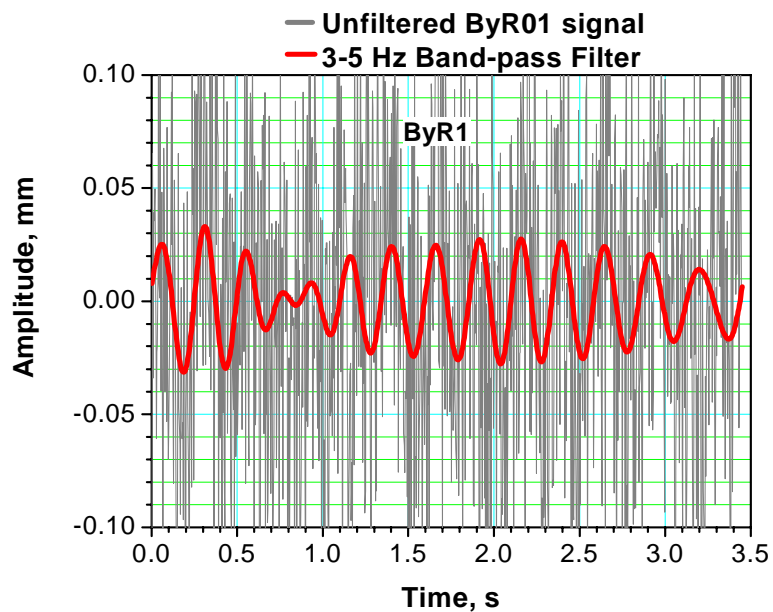

Fig. 18a. Unfiltered and 3-5 Hz band-pass filtered BYR01 signals.

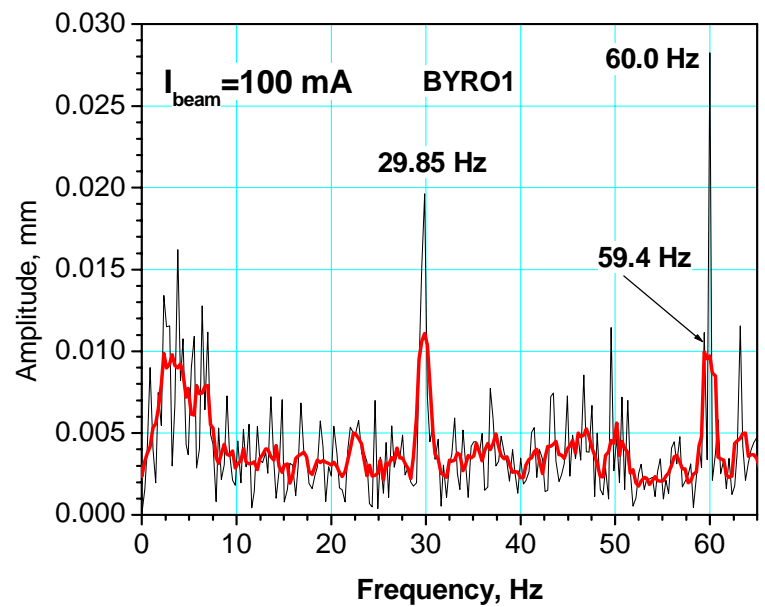

Fig.18b. FFT transformation of the BPM BYR01 signal. Red curve indicates averaging over 5 points. 
The figures indicating the low-frequency oscillations in ByR01 signals have components in the range of 3-7 Hz. The $3.9 \mathrm{~Hz}$ harmonic can be explained as the deviations in the Pelletron energy caused by oscillation in the stabilizing feedback circuitry in which affects the terminal shell vibration, as shown in Section 6. From estimations with formula (2.3) for the dispersion value of $130 \mathrm{~cm}$ the amplitude of the Pelletron voltage deviations is $\sim 100 \mathrm{~V}$. This value corresponds to the upper limit given by (4.2). Comparison of the FFT spectrum with spectra plotted in Fig. 14 shows that the voltage deviations caused by the terminal shell motion are noticeable at significant dispersion regions but in the cooling section they have no affect on the beam motion.

\section{Measurements of mechanical vibrations in the Pelletron terminal}

As mentioned in Section 3, vibrations in the Pelletron terminal were measured by three types of sensors. A piezo vibration meter, velocimeters and geophones were used with detailed measurements made with the latter only.

The piezo vibration meter measurements provided estimations of vibration amplitudes in various locations. Measured displacements were about $10 \mu \mathrm{m}$ at the surface of the terminal separation box, $20 \mu \mathrm{m}$ at the gun flange (marked 4 in Fig. 5), and $30 \mu \mathrm{m}$ at the gun lens body (marked 3 in Fig. 5). The highest level of vibrations was found at the gun ion pump (5 in Fig. 5), 90-125 $\mu \mathrm{m}$.

The next set of measurements was performed with two velocimeters located at the upper separation box (terminal). The velocimeters showed good resolution while measuring the background vibrations. Turning the shaft motor on increased the level of vibrations by two orders of magnitude, saturating the velocimeters. To avoid the saturation, spectra were taken with the attenuation of 10 and a frequency cutoff of $20 \mathrm{~Hz}$ (Fig. 19). In the horizontal plane, a $30 \mathrm{~Hz}$ harmonic is the largest, with 20 and $40 \mathrm{~Hz}$ amplitudes more than 10 times lower. In the vertical plane, a $30 \mathrm{~Hz}$ harmonic dominates as well; its amplitude is approximately 5 times lower than in the horizontal plane.

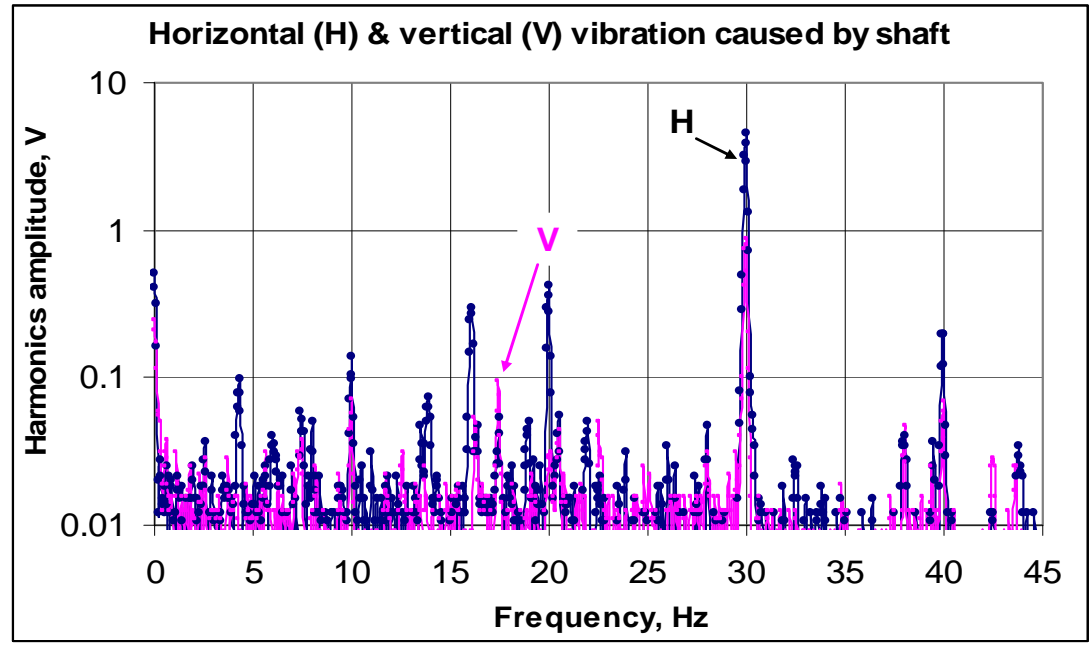

Fig. 19. Vibrations spectra measured by velocimeters. The calibration curve is in Fig.7.

The most detailed measurements of vibrations were done with geophones. In these measurements, the shaft was loaded by operating the solenoidal lenses in the separation boxes. Vibrations caused by the revolving shaft and by the running chain were 
measured separately. Corresponding contributions to displacements of the upper separation box are shown in Figs. 20 and 21. Comparison of displacements calculated from the spectra measured using velocimeters with those measured by geophones demonstrates qualitative coincidence.

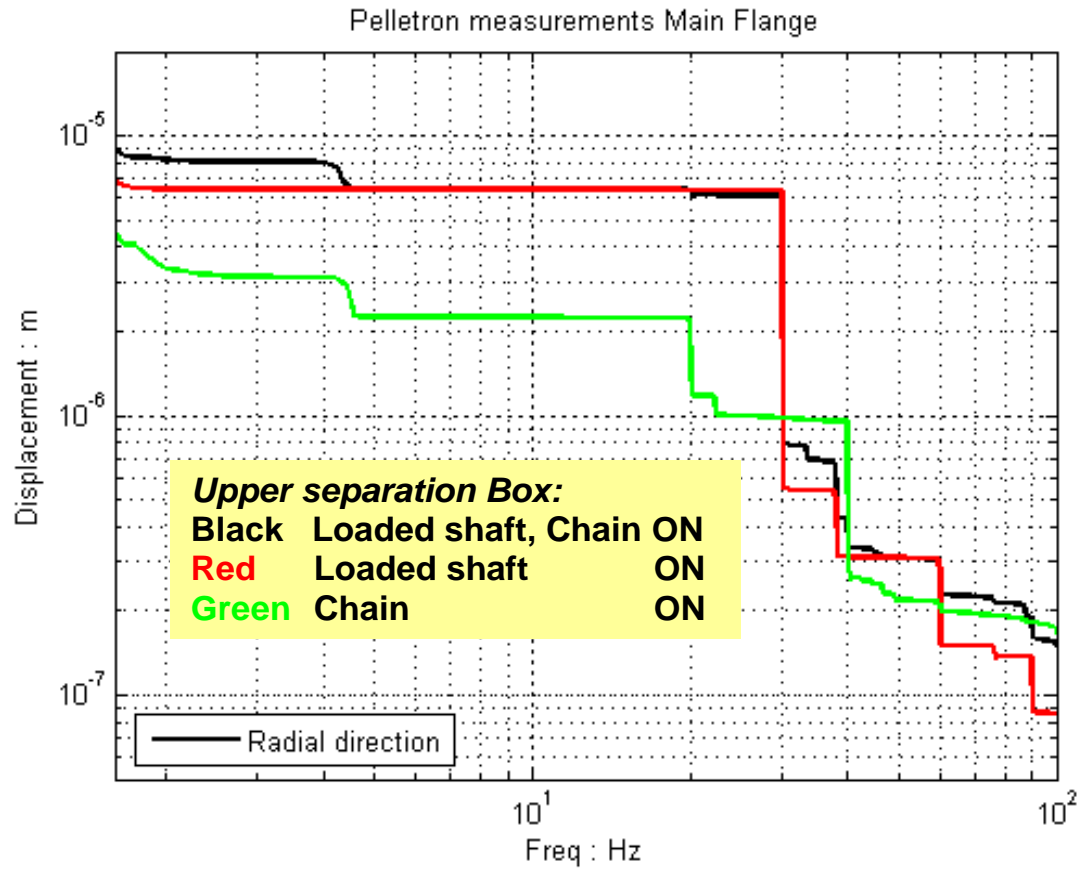

Fig. 20. Displacements of the terminal separation box in the horizontal plane caused by different sources.

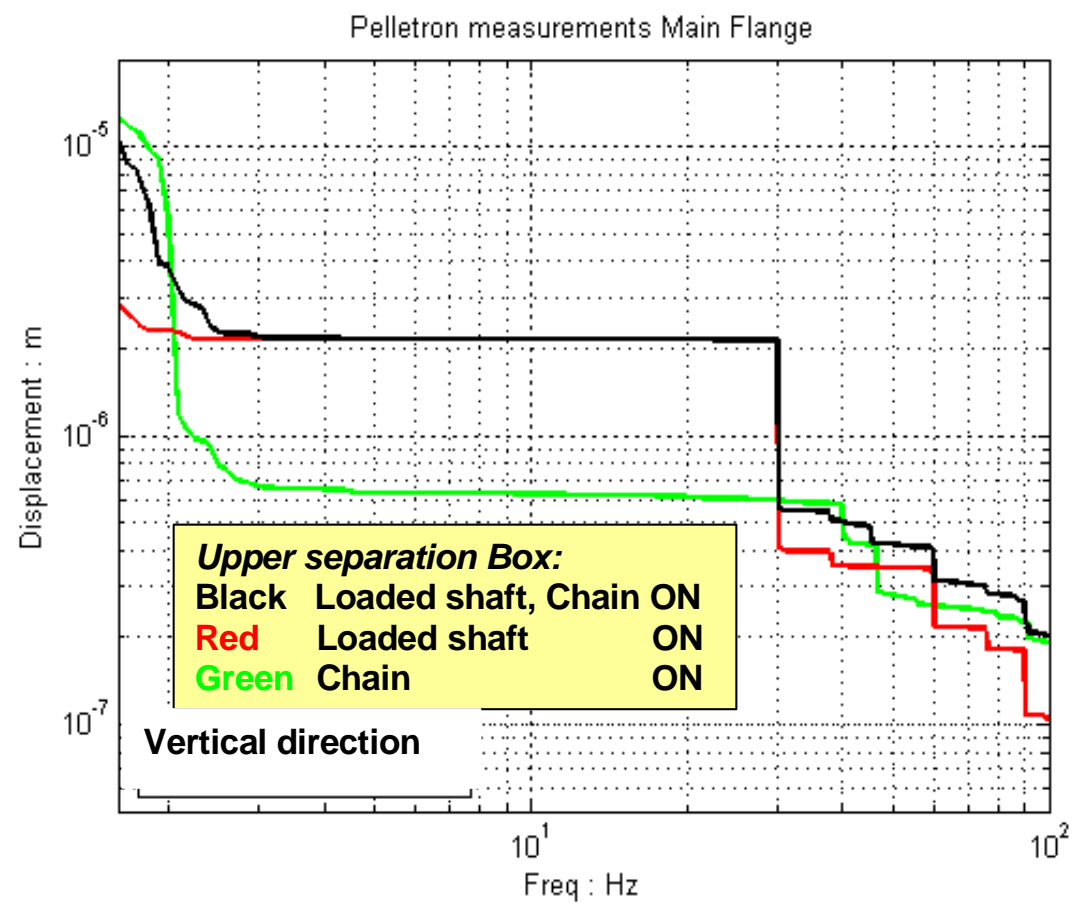

Fig. 21. Displacements of the terminal separation box in the vertical plane caused by different sources. 
The maximum displacement amplitude in the horizontal plane is about $8 \mu \mathrm{m}$. The main source of vibration was found to be the revolving shaft. The major component of resulting oscillations has a frequency of $\approx 30 \mathrm{~Hz}$, which was identified as the motor revolution frequency (see Table 2); the second harmonic was observable as well.

The chains excited oscillations with frequencies of 4.46, 19.9, and $39.85 \mathrm{~Hz}$ (Fig. 22). The latter two are close to the chain motor rotation frequency and its second harmonic. The $4.46 \mathrm{~Hz}$ component is most likely related to a transverse oscillation of the chains. The chain between sheaves can be modeled by a string clamped on its ends. In this case the lowest harmonic of the transverse oscillation of the chain is:

$$
f_{t}=\frac{v}{l} \approx 5 \mathrm{~Hz}
$$

were $v$ is the nominal chain speed, and $l$ is the distance between sheaves (see Table 2). Detailed profiles of the low-frequency oscillations are plotted in Fig. 23. One can see that this oscillation is more noticeable in the plane of the chain (measured with the tangential sensor). The measured $Q$-factor value of the $4.46 \mathrm{~Hz}$ oscillation is $\sim 45(Q=f / \Delta f)$.

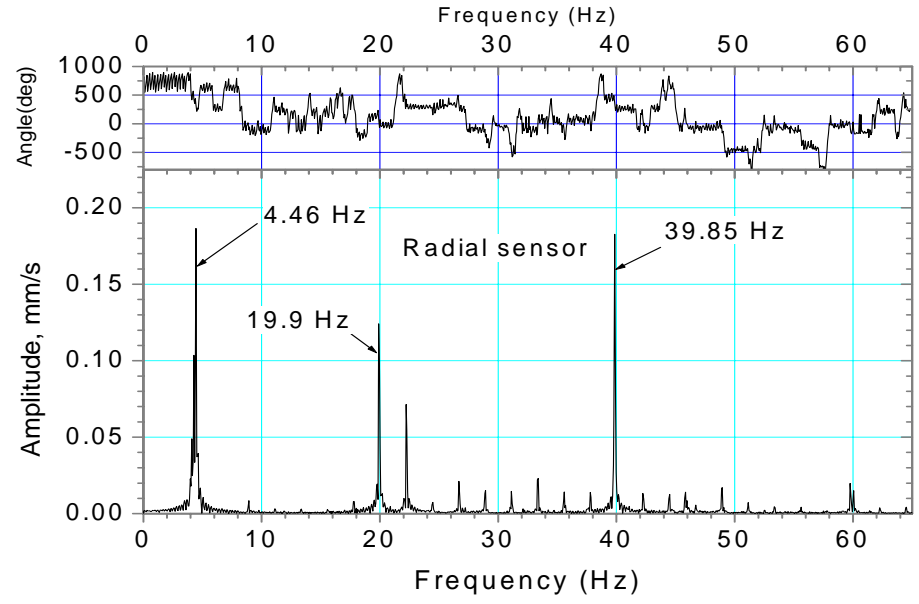

Fig. 22. Spectrum of terminal separation box oscillations caused by the chain. Measurements are made with the radial sensor.

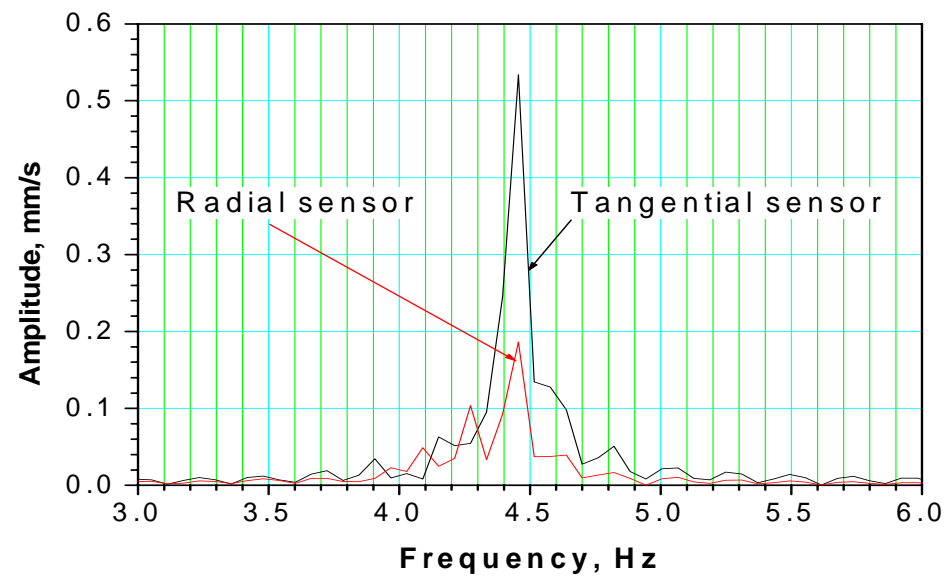

Fig. 23. 4.46-Hz oscillation caused by the running chain. 
Similar measurements were performed with the sensor assembly placed at the gun flange and at the top of the gun lens (marks 4 and 3 at Fig. 5). The maximum displacements were found equal to $6 \mu \mathrm{m}$ and $9 \mu \mathrm{m}$, respectively (Figs. 24 and 25).

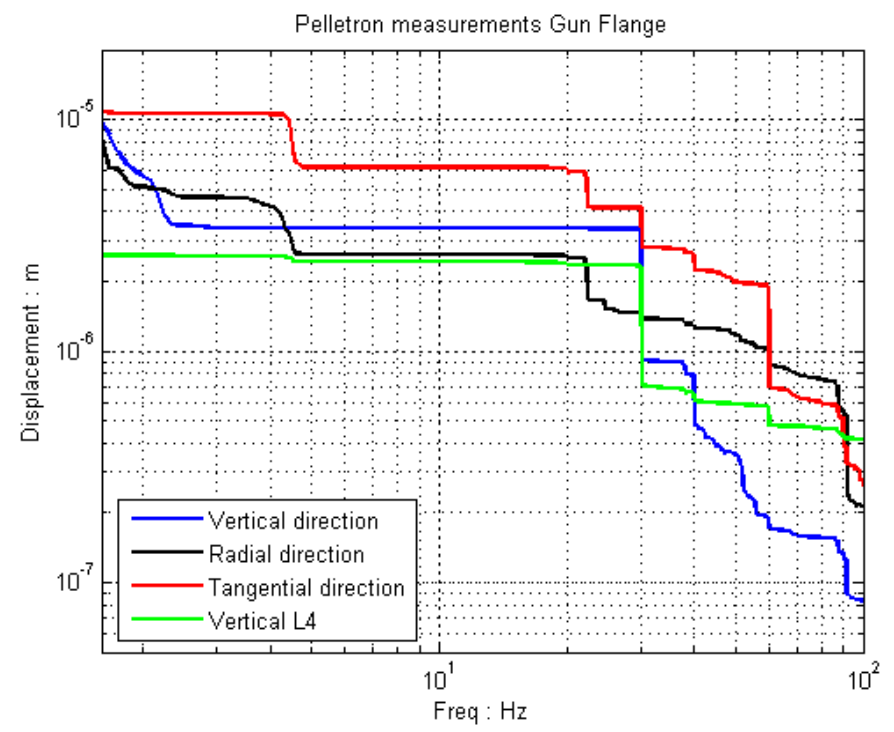

Fig. 24. Displacements of the gun flange for oscillations caused by the shaft and chain.

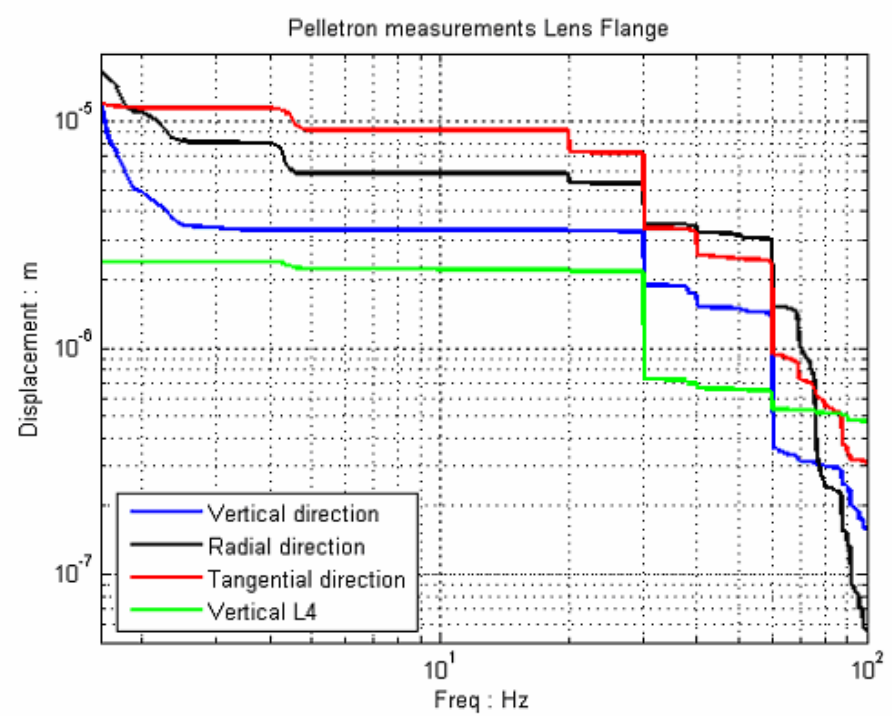

Fig. 25. Displacements of the gun lens for oscillations caused by the shaft and chain.

\section{CPO monitor signals}

Motion of the terminal shell can distort measurements of the voltage fluctuations and affect operation of the Pelletron voltage stabilization circuitry. An attempt to analyze these effects was done by studying the CPO signals with a Pelletron voltage of $3.5 \mathrm{MV}$ which corresponded to operating condition.

There are two capacitor pick-ups (CPO) located on the Pelletron tank wall for the purpose of monitoring terminal voltage ripple. Time and frequency domain analysis were done on the CPO signals in an attempt to correlate observed beam motion in the transport 
line with signals on the terminal. The CPO signals result from changing electric fields, therefore the CPO signals reveal both voltage ripple on the terminal as well as lateral motion of the terminal when the terminal is at operating voltage, in this case $3.5 \mathrm{MV}$.

This section discusses the signals observed on the CPO monitors. Analyses of time and frequency domain signals reveal there is terminal motion from the motor/generator shaft and charging chain rotation and terminal voltage ripple caused by instability in the voltage regulation system.

\subsection{CPO Specifications}

The each of the two CPO pick-ups mounted on the tank are input to an amplifier box located on the upper catwalk. The gains of the resulting output signals are calibrated. The outputs of the CPO amplifiers are routed to the TPS chassis where the signals are buffered, summed together and brought to the chassis rear panel for monitoring. Although different from the manufacturer's claimed calibration, the measured specifications for the CPO signals are:

Each CPO signal gain: $\quad$ +/-1 V per $+/-1000 \mathrm{~V}$ of terminal voltage ripple.

CPO bandwidth: $\quad 1 \mathrm{~Hz}$ to $10 \mathrm{KHz}$.

CPO sum signal gain: $\quad+/-1 \mathrm{~V}$ per $+/-100 \mathrm{~V}$ of terminal voltage ripple;

$$
\mathrm{Sum}=(\mathrm{CPO} 1+\mathrm{CPO} 2) \times 5 \text {. }
$$

\subsection{Terminal Shell Motion from Chains and Shaft}

The two CPO monitors are located on opposite sides of the tank with the design intent of canceling out the terminal motion by summing the two. In practice, motions of the terminal shell caused by terminal vibrations are not always out of phase between CPO- 1 and CPO- 2 and, thus, are not cancelled in the sum signal. This is revealed in the time domain plots of Fig. 26, 28 and 29. The low frequencies in these plots are in phase at CPO-1 and CPO-2, yet they are due to motion, Fig. 26, from only the chains and, mainly, from the running chains and the rotating shaft, Fig. 28. These plots superimpose the result of applying narrow band filters to better reveal frequencies of interest.

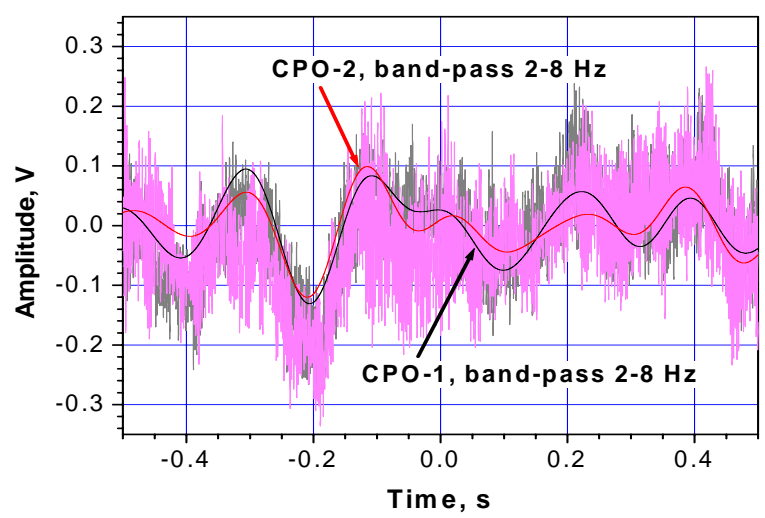

Fig. 26. Unfiltered and filtered CPO-1 and CPO-2 signals. Chains are on; shaft is stopped; feedback is off.

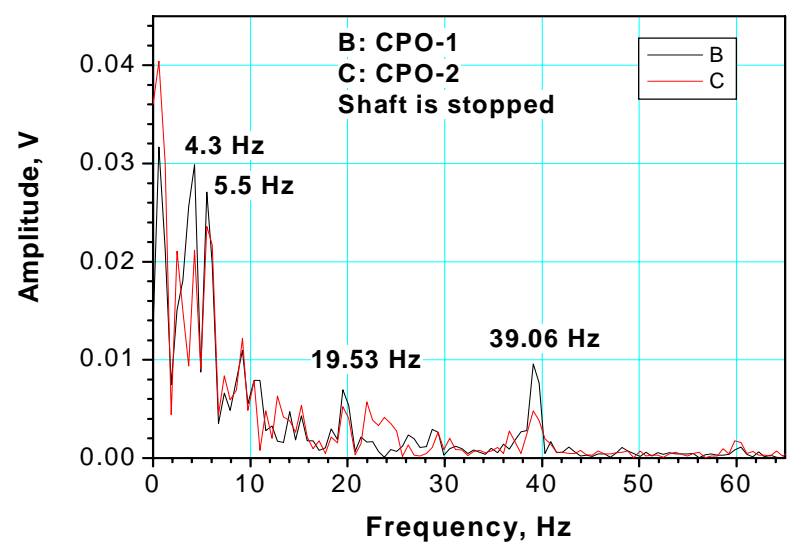

Fig. 27. CPO spectra with stopped shaft. Chains are on; feedback is off. 
Chain motion is observable specifically in Fig. 26 and 27, where the regulation loop and shaft are turned off. Also observable in the FFT of Fig. 27 is the chain motor revolution rate of $19.5 \mathrm{~Hz}$ and its second harmonic at $39 \mathrm{~Hz}$. The motor is an $1150 \mathrm{RPM}$, 7.5 HP induction type, which explains the frequency just under $20 \mathrm{~Hz}$. Their effect, however, is negligible. Motion from the chains, itself, is calculated and observed at $\approx 4$ $\mathrm{Hz}$.

The shaft motor is a $1760 \mathrm{RPM}, 25 \mathrm{HP}$ induction type responsible for the $29 \mathrm{~Hz}$. Figs. 30, 31 are the plots showing the time and FFT plots, respectively. The dominant frequency of $29 \mathrm{~Hz}$ was always observed in phase at CPO-1 and CPO-2, Figs. 28-30.

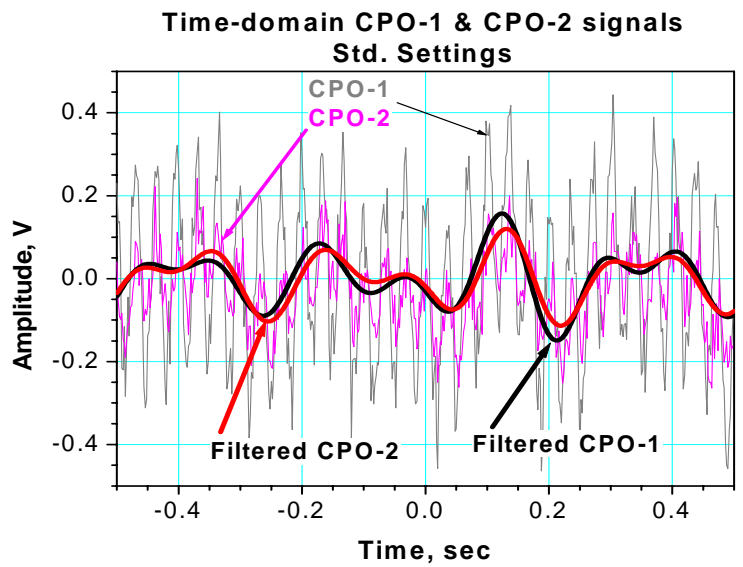

Fig. 28. In-phase deviation of CPO-1 and CPO-2 signals caused by shaft and chains. Bold lines show signals filtered with the bandwidth of 2-8 Hz.

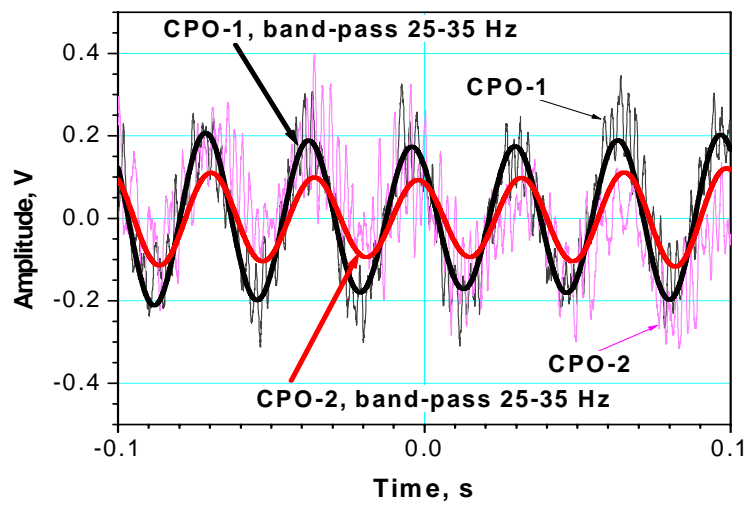

Fig. 30. CPO-1 and CPO-2 signals caused by revolving shaft and running chains with and without the band-pass filtering.

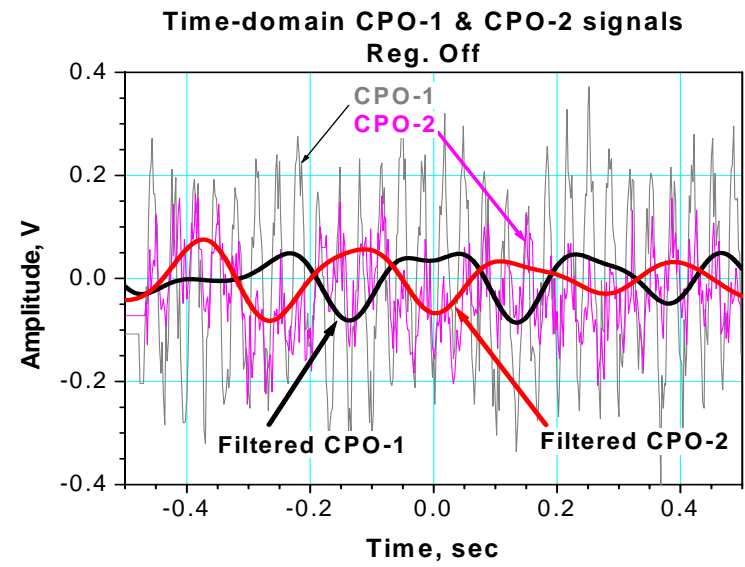

Fig. 29. Out-of-phase deviations of CPO-1 and CPO-2 signals caused by shaft and chains. Bold lines show filtering with the bandwidth of 2-8 Hz.

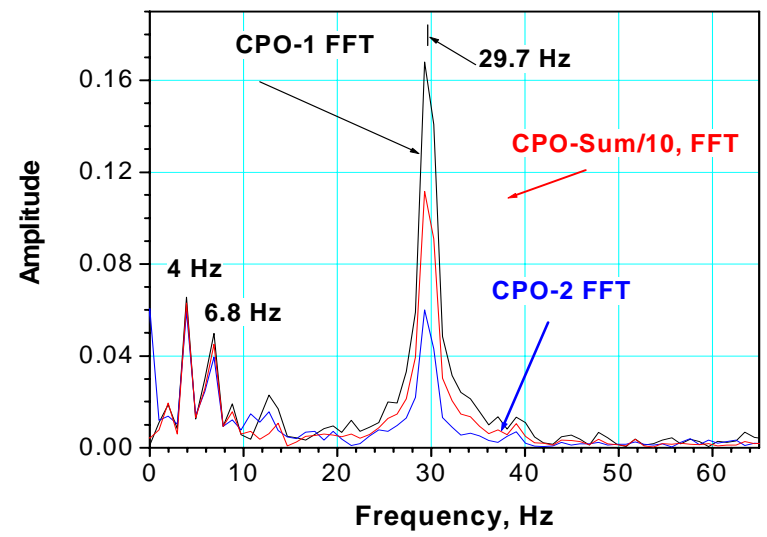

Fig. 31. FFT spectra of the CPO signal with revolving shaft and running chains

\subsection{Variations with time}

It must be noted that there was considerable variations of relative amplitudes on the two pick-ups with time. For example, the dominant $\sim 30 \mathrm{~Hz}$ signals on CPO-1 and CPO-2 changed continuously relative to each other from 1:1 to 1:3 within a timeframe of 
roughly 30 seconds. This is true for signals at other frequencies as well, but we did not document the range of signal amplitudes at each frequency. Data was taken as snapshots in time and do not represent any sort of average.

Frequency components were not always observed in phase with each other at the two CPOs, Figs. 28, 29. Both plots are with the same conditions, only on second the regulation was turned off. At two different times, the phases of the low frequencies were different at CPO-1 and CPO-2.

By estimation, an observation of 0.15 Volts at one CPO corresponds to $40 \mu \mathrm{m}$ of terminal shell motion, with $3.5 \mathrm{MV}$ on the terminal, the known gap of 36 inches between the terminal shell and the CPO pickup and the CPO gain of 1000. This corresponds to more strong oscillations of the terminal shell in comparison with terminal, Figs. 20, 21. Moreover the shell has additional modes of oscillations caused by operation of the chains and the shaft and observable at low frequencies. In-phase oscillations, Fig. 28, indicate this. Comparison of Figs. 22 and 31 show significantly broader spectral lines for the lowfrequency shell oscillations by the operating Pelletron. It points to damping of the transverse oscillations caused by running the chains in high-pressure $\mathrm{SF}_{6}$ gas.

\subsection{Regulation Loop Voltage Ripple}

Fig. 32 shows the effect of varying the terminal regulation loop gain. It is an FFT of the CPO sum signal. Frequencies in the 5-6 Hz range varied significantly, while $4 \mathrm{~Hz}$ and $29 \mathrm{~Hz}$ did not. By comparison, curve B is with regulation turned off-it is from data of Fig. 29.

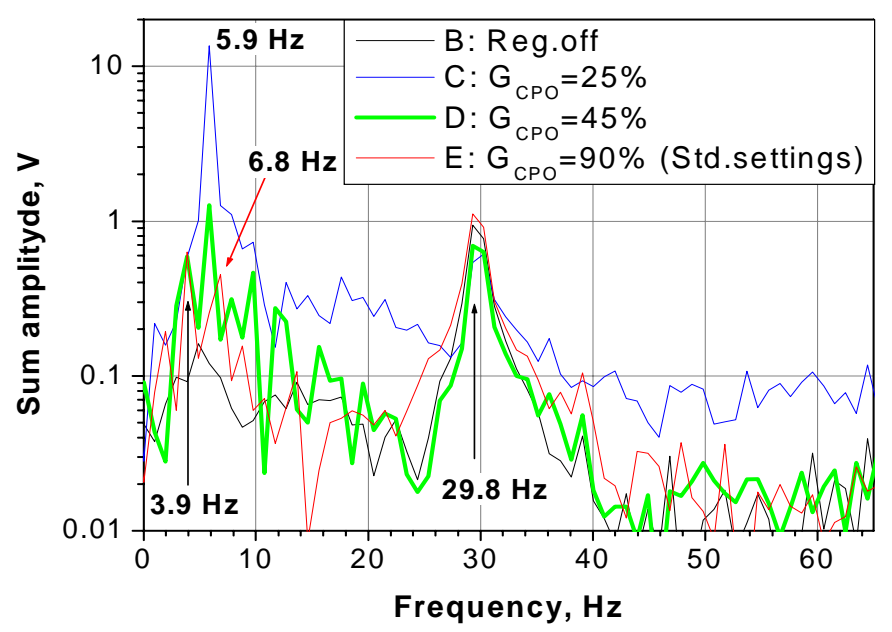

Fig. 32. CPO spectra at various feedback gains. The presented signals are a hardware sum of signals from two individual CPOs. Small amplitude of the low-frequency oscillations shown by curve B was caused by out-of-phase oscillations in CPO-1 and CPO-2 signals, Fig. 29.

At the frequency of $\sim 5-6 \mathrm{~Hz}$, the CPO signals changed significantly at varied feedback loop gain setting, while $30-\mathrm{Hz}$ components were approximately the same, being outside of the loop bandwidth. A 4-Hz component of the oscillation presented by curves $\mathrm{C}, \mathrm{D}$, and $\mathrm{E}$ also has no dependence on the feedback loop gain. So 5-6 Hz component is, perhaps, the parasitic oscillation of the feedback loop. 
In addition, note the time-to-time variability in the amplitude of the $6.8 \mathrm{~Hz}$ oscillations. Fig. 33 presents FFT spectra of the CPO sum signals measured by different accelerated currents at different times. One can see stable enough amplitude of $4 \mathrm{~Hz}$ and 29.7 Hz harmonics and noticeable difference in amplitudes of $6.8 \mathrm{~Hz}$ harmonics.

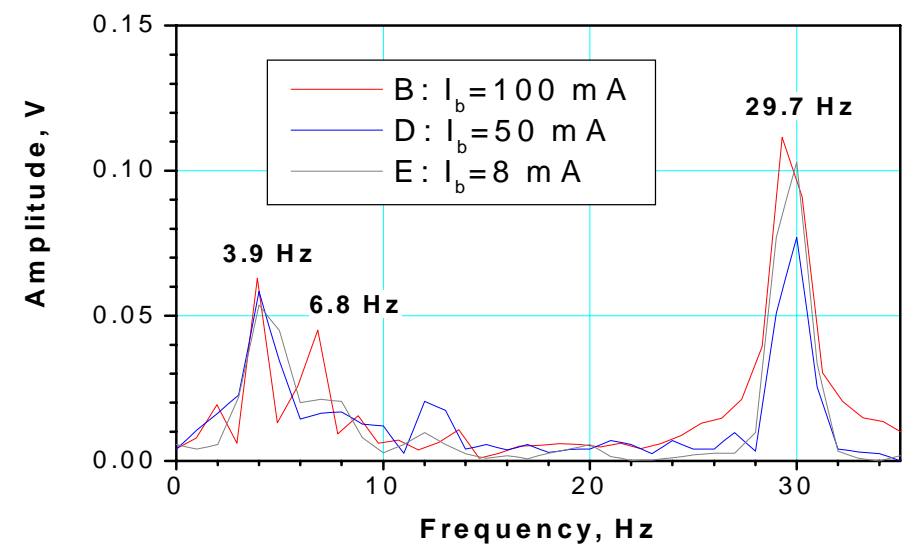

Fig. 33. CPO sum spectra measured at different time with different accelerated beam current.

Note that frequencies of terminal motion must not be inside the bandwidth of the regulation loop. Otherwise, these signals will result in the loop responding by applying a voltage to the terminal in an attempt to reduce them. In our case, there seems to be an undesirable effect from loop instability, although it may be at a level that is not significant. At DC, the loop should reduce, or eliminate, the effect of beam loading on the terminal, so it is not clear that turning off the loop is the right thing to do.

\section{Discussion}

Let us correlate the results from three set of measurements: mechanical vibrations, electric field at the tank surface (CPO signals), and beam oscillations.

\subsection{Beam oscillations}

One of major components of the beam oscillations spectrum, $29.8 \mathrm{~Hz}$ line, has, with precision of measurements, the same frequency as the shaft motor rotation; also, the beam analysis traces the origin of the component to the Pelletron. Taking into account that the same frequency is found in the CPO spectra and in vibration measurements, the vibration is a possible source of excitation of the beam oscillations. It is difficult to calculate effect of vibrations on the beam displacements without an exact knowledge of the source. Also, optics simulations in the acceleration tube were not very reliable. Approximately, the influence of the beam motion inside the Pelletron $\chi_{P}$ on the beam motion inside the cooling section $\chi_{C}$ can be estimated by a ratio of the corresponding beam radii:

$$
\chi_{C} / \chi_{P} \cong a_{C} / a_{P}
$$

Assuming the beam diameter in the cooling section of $12 \mathrm{~mm}$, and the cathode diameter of $7.6 \mathrm{~mm}$, the corresponding amplification factor is 1.6. The maximum 
oscillation amplitude at this frequency found in the cooling section is $\sim 50$ microns. Therefore, the oscillations can be explained by vibrations with amplitude $\sim 30$ microns. The value is much higher than the measured values of vibration amplitudes at this frequency, 2 - $9 \mu \mathrm{m}$ (Figs. 20, 24, 25) and comparable with the estimation of the terminal shell vibration from CPO spectra. One possible explanation is that the vibration measurements were not made at the element, determining the beam oscillation amplitude. One can speculate that the gun cathode could be the element. As it was pointed out in Section 5, maximum amplitude of vibrations in the band of $8-100 \mathrm{~Hz}$, measured with the piezo vibration meter, was found at the gun ion pump. The gun cathode is mounted on the opposite side of the gun flange with a comparable lever (Fig. 5) and might experience in similar vibrations.

Latest measurements of the stray magnetic fields caused by operation the chains and shaft motors indicate very low level of the alternative magnetic field; the magnetomotive force is $\sim 1.9 \mathrm{mG} \cdot \mathrm{m}$. Corresponding lower harmonic of the magnetic field has frequency of $60 \mathrm{~Hz}$. Estimated bending angle for the accelerated beam is $\leq 15$ $\mu \mathrm{rad}$ and can't make any obstacle for electron cooling.

The line corresponding to the second harmonic of the shaft rotation frequency, $59.5 \mathrm{~Hz}$, has similar strength in the BPM spectra and also was traced to the Pelletron. While the component with a similar frequency exists in the vibration spectra, there is no quantitative agreement as well. As it was mention above, the $60 \mathrm{~Hz}$ component of the beam motion seems to be not related to the Pelletron.

A $20 \mathrm{~Hz}$ line is clearly seen at most BPMs with amplitude several times lower than the main peaks. This weak component, present in the CPO spectra, Fig. 27, is excited by the running chains, but with the revolving shaft (by operating the Pelletron) it is not clearly seen, Fig. 31. This agrees with corresponding minimal displacement in the terminal vibration spectra (Fig. 20). On the other hand, there are $20 \mathrm{~Hz}$ signals in the gun lens and the gun flange spectra (Figs. 24 and 25), and the line can be correlated with the rotation frequency of the chain motor.

Low frequency components, which have comparable or even larger values than the $30 \mathrm{~Hz}$ ones, are not present in the beam motion spectra, only the weak peaks are seen in places having significant dispersion, Fig. 18. It is necessary to remember, that the vibration measurements were performed with the Pelletron tank open to atmosphere, while the CPO and beam motion spectra were taken with the tank filled with a dense heavy insulating gas $\left(70\right.$ psi of $\left.\mathrm{SF}_{6}\right)$. The gas may significantly damp some modes. For example, one can argue that the chain transverse oscillations, which can explain the 4.46 $\mathrm{Hz}$ frequency line in the vibration spectra (Fig. 22), are dramatically suppressed in this dense media.

At the measured level, the electron beam oscillations are not considered a big concern. The r.m.s. value of the oscillations of $60 \mu \mathrm{m}$ corresponds to $30 \mu \mathrm{rad}$ contribution to the total angle budget of the electron beam, while the upper acceptable boundary for the budget is $200 \mu \mathrm{rad}$. Therefore, lowering the vibration level is not critically important. While reassembling the Pelletron in its permanent location at the Recycler ring, in the MI-31 building, only one improvement is planned, namely, securing the gun ion pump with respect to the deck separation box to reduce its oscillation.

A feedback system for stabilization of the beam position in the cooling section in the bandwidth up to $100 \mathrm{~Hz}$ is under development. The system will adjust currents in the 
dipole correctors upstream of the cooling section using information from the cooling section BPMs. Along with its main purpose the system should significantly reduce other oscillations as well, such as, counteracting Main Injector fringe fields and possible $60 \mathrm{~Hz}$ power grid fields.

\subsection{Energy fluctuation}

Analysis of BPM readings in a high-dispersion region (see Part 4.3) gives an upper limit for energy fluctuations at all main frequencies $\leq 100 \mathrm{eV}$.

For electron cooling to be effective, the r.m.s. energy fluctuations should be kept below $1 \cdot 10^{-4}$ of the total energy. Corresponding acceptable level of the terminal voltage fluctuations, $350 \mathrm{~V}$, is well above.

\section{Summary}

Three different types of measurements were performed. The beam motion was measured with the BPM system; variations of the electric field at the tank surface were recorded with capacitive pickups; and vibrations were recorded at several locations in the Pelletron terminal with the Pelletron tank filled with air at atmospheric pressure. The dominant frequency of $29.8 \mathrm{~Hz}$, corresponding to the shaft rotation frequency, was found in all measurement sets. This correlation makes it very probable that a significant portion of the beam oscillations in the cooling section is caused by vibration of optics elements in the acceleration tube, though no direct quantitative agreement was found.

\section{Acknowledgments}

The authors are thankful to the entire Recycler Electron Cooling group for help in performing these measurements and showing interest in the work. We acknowledge fruitful participation of $\mathrm{H}$. Pfeffer in CPO measurements and in the discussions of the results. The velocimeters were provided by V. Shiltsev who helped with setting them up. The drawings were prepared by S. Wesseln.

\section{References}

1. Prospectus for an electron cooling system for the Recycler. By J.A. MacLachlan, A. Burov, A.C. Crawford, T. Kroc, S. Nagaitsev, C. Schmidt, A. Sharapa, A. Shemyakin, A.Warner, Proc. of $4^{\text {th }}$ Workshop on the Medium Energy Electron Cooling MEEC-98, edited by I. Meshkov, Dubna, 1999, pp. 25-113. Available at: http://library.fnal.gov/archive/test-tm/2000/fermilab-tm-2061.shtml .

2. Pelletrons are manufactured by the National Electrostatics Corporation, www.pelletron.com .

3. Test of a full-scale prototype of the Fermilab cooler, by A. Shemyakin, et al., to be published in Proc. of APAC'04, Korea, March 22-26, 2004.

4. V. Lebedev, "OptiM - Computer code for linear and non-linear optics calculations," http://www-bdnew.fnal.gov/pbar/organizationalchart/lebedev/OptiM/optim.htm . 